\title{
Utilization, effect, and benefit of the individualized Meeting Centers Support Program for people with dementia and caregivers
}

This article was published in the following Dove Press journal:

Clinical Interventions in Aging

\author{
Rose-Marie Dröes $\mathbb{D}^{1,2}$ \\ Annelies van Rijn' \\ Eline Rus ${ }^{3}$ \\ Seghoslène Dacier ${ }^{4}$ \\ Franka Meiland (iD) $1,4,5$ \\ 'Department of Psychiatry, Amsterdam \\ University Medical Centers, Location \\ Vumc, Amsterdam Public Health \\ Research Institute, Amsterdam, The \\ Netherlands; ${ }^{2}$ Department of Research \\ and Innovation, Regional Mental Health \\ Organization Ggzingeest, Amsterdam, \\ The Netherlands; ${ }^{3}$ Department of \\ Clinical Pyschology, Faculty of Behavior \\ and Movement Sciences, VU University, \\ Amsterdam, The Netherlands; \\ ${ }^{4}$ Department of Neuropsychology, \\ Faculty of Behavior and Movement \\ Sciences, VU University, Amsterdam, The \\ Netherlands; ${ }^{5}$ Gerion, Department of \\ General Practice and Elderly Care \\ Medicine, Amsterdam University Medical \\ Centers, Location Vumc, Amsterdam, \\ The Netherlands
}

Correspondence: Rose-Marie Dröes Department of Psychiatry, Amsterdam UMC, location VUmc, GGZ-inGeest Dienst onderzoek en innovatie,

Oldenaller I, I08I HJ Amsterdam, Postbox 74077, Amsterdam 1070 BB, the Netherlands

Tel +3I 207885454

Email rm.droes@amsterdamumc.nl
Purpose: There are few interventions on an individual basis to support community-dwelling people with dementia to continue to fulfill their potential in society and to support their informal caregivers via e-Health. This study explored the effectiveness of the individualized Meeting Centers Support Program (iMCSP) consisting of DemenTalent (people with dementia work as volunteers in a society based on their talents), Dementelcoach (telephone coaching), and STAR e-Learning for caregivers, compared to regular MCSP and No day care support.

Method: An explorative randomized controlled trial with pre/post measurements (M0-M6) and two groups (iMCSP and regular MCSP). In addition, a comparison was made between iMCSP and a reference No day care control group. Standardized questionnaires were administered on self-esteem, neuropsychiatric symptoms, experienced autonomy and quality of life of the person with dementia, and on caregiver's sense of competence, quality of life, and happiness.

Results: The iMCSP interventions resulted in a broader group of participants utilizing the Meeting Centers. Compared to regular MCSP, DemenTalent had a moderate positive effect on neuropsychiatric symptoms, which also proved less severe. Positive affect of participants improved within the DemenTalent and regular MCSP group after six months. Caregivers of DemenTalent participants experienced less emotional impact of neuropsychiatric symptoms. No differences were found in experienced burden, sense of competence, or quality of life in caregivers using iMCSP or regular MCSP. Compared to those receiving No day care support, caregivers of DemenTalent participants and caregivers using Dementelcoach or STAR eLearning proved happier. Post-hoc analyses, accounting for potential between-group differences in outcome measures at baseline, generally showed results in the same direction. People with dementia and caregivers highly appreciated iMCSP and regular MCSP.

Conclusion: iMCSP can be effectively applied as alternative or additional support via regular Meeting Centers for people with dementia and caregivers who prefer individualized activities/ support. DemenTalent decreased the severity of neuropsychiatric symptoms of people with dementia and emotional burden of caregivers. All iMCSP interventions tended to result in caregivers being happier compared to those receiving no support. Larger-scale studies are needed to investigate the effect of iMCSP on other domains of quality of life of participants. Keywords: individualized support, DemenTalent, telephone coaching, e-Learning, neuropsychiatric symptoms, emotional burden caregivers

\section{Introduction}

Dementia has major consequences for people with dementia themselves and for their loved ones. Research shows that $70 \%$ of the people with dementia stop doing activities due to a lack of self-confidence and 40\% hardly ever leave the house. ${ }^{1}$ 
Many studies highlight the burden experienced by family caregivers. ${ }^{2,3}$ The recently published Dutch Dementia Caregiver monitor showed that $12.5 \%$ feel overburdened, $52 \%$ moderately to heavily burdened, and more than $50 \%$ have general and mental health problems. ${ }^{4}$ Isolation and loneliness are major issues for those living with dementia and caregivers. ${ }^{4,5}$

Studies on the needs of people with dementia living at home highlight social contact as one of the most important needs, besides the need for greater self-esteem, being useful, and enjoyable and meaningful activities. ${ }^{4,6,7}$ Fulfilling these needs, as well as the need for self-determination and being accepted as you are, in an individualized and personcentered way proves critical for the quality of life of people with mild-to-moderate dementia. ${ }^{8}$ Informal caregivers indicate that they mainly need information, practical, and emotional support, eg from home care and a case manager, and that meaningful daytime activities are especially important for the person with dementia., ${ }^{4,7}$ Timely information (psychoeducation) and the use of care and support services can help people with dementia maintain a good quality of life and prevent informal caregivers from becoming overburdened. Practice and research, however, show that people often postpone asking for help. Reasons for this include the taboo and stigma of dementia, resistance of the person with dementia, feelings of guilt of the informal caregiver, and the lack of matching of services with personal needs and preferences. ${ }^{7,9}$ In addition, it is difficult for people to find the right care offer due to the fragmentation of the care and support offer.

The Meeting Centers Support Program (MCSP) for people with dementia and their informal caregivers tries to tackle these problems by offering combined support for both by a small professional staff and volunteers at an easy accessible, socially integrated location in the neighborhood. People with dementia are offered meaningful recreational, creative, and therapeutic activities in the Meeting Center (their "club"), the caregivers can attend informative meetings and discussion groups, and both can participate in social activities and utilize individual consultation. This combined form of support is highly appreciated by people with dementia and informal caregivers, and both benefit from it. This is evident not only from the great increase in the number of Meeting Centers over the past 25 years (there are now 163 centers across the Netherlands and about 25 in other countries in and outside Europe) but also from repeated scientific research. ${ }^{10-16}$ Several studies have shown that the users of the Meeting Centers are almost unanimously (very) satisfied with the offer ${ }^{13,17}$ and that compared to regular day care, the participants with dementia using MCSP have fewer behavioral and mood problems and higher self-esteem, ${ }^{10,11,15}$ their informal caregivers feel more competent, nursing home admission is postponed, ${ }^{12}$ and lonely informal caregivers have fewer health complaints. ${ }^{14}$ However, recent figures ${ }^{18}$ show that nationwide only around $10-20 \%$ of the people with dementia (and informal caregivers) in the Netherlands use different forms of organized daytime activities ( $7 \%$ of those use indicated day care). ${ }^{1,18}$ To ensure that more people can receive appropriate psychosocial support it is therefore important that, in addition to day care, alternative forms of activities and support become available that better meet the varied individual needs of people living with dementia and their caregivers in different stages of the disease.

In order to meet the variety of individual needs and preferences of people with dementia and their informal caregivers, innovative interventions have been developed and offered in the last decade. Three of these interventions, which have also been assessed very positively by people with dementia and caregivers themselves, are DemenTalent, Dementelcoach, ${ }^{19}$ and STAR e-Learning. ${ }^{20,21}$

The DemenTalent volunteer project meets the needs of people with dementia for autonomy, being useful by contributing to society as a volunteer and to maintain dignity. Practice shows that people feel they have value again and that this promotes social participation and more independent functioning. The project also contributes to a more positive image of dementia and counters stigmatization. ${ }^{22}$

Dementelcoach offers informal caregivers tailored telephone support for the problems they experience as caregivers, in which a great deal of attention is paid to the burden experienced by caregivers and methods for reducing stress and transgressive behavior toward the person with dementia. A controlled trial showed that the coaching increased caregivers' feelings of competence, decreased psychosomatic complaints when applied in combination with day care for the person with dementia, and was highly appreciated by caregivers. ${ }^{19}$ Another recent study showed that telecoaching diminished depression in caregivers and improved self-efficacy. ${ }^{23}$

STAR e-Learning is a course recently developed in a European project and accessible via the Internet (www. startraining.eu). The course consists of 8 modules for informal caregivers and volunteers (in addition to 6 advanced modules for professional caregivers) and is aimed at 
increasing their knowledge and skills in order to provide the person with dementia with person-oriented care, to deal with behavioral changes, to prevent or decrease neuropsychiatric problems, and to take good care of themselves as caregiver. ${ }^{20}$ STAR education was positively evaluated in a randomized controlled trial (RCT) in the Netherlands and the UK, both on user-friendliness and usefulness. Informal caregivers who had completed the STAR course proved to offer more person-oriented care and to have more empathy for the person with dementia. ${ }^{21}$

In the individualized Meeting Centers Support Program (iMCSP) project (2016-2019), we investigated the implementation of these three interventions (DemenTalent, Dementelcoach, and STAR e-Learning) in existing Meeting Centers for people with dementia and their caregivers. ${ }^{22}$ The expectation was that, by adding these individualized, personoriented interventions parallel to the regular group-oriented support program offered by the Meeting Centers to date, the variation in people's needs and preferences could be better addressed, so that more people with dementia and informal caregivers would make use of the activities and support. The implementation study, on which we reported elsewhere, showed that different stakeholders (such as Meeting Centers' personnel, Alzheimer Association, local government) indeed experienced the new iMCSP interventions as added value to the regular MCSP. ${ }^{22}$ In addition, we evaluated the characteristics of participants of iMCSP compared to regular MCSP and No day care users, and the impact of the individualized interventions on people with dementia and caregivers compared to regular group-oriented MCSP and compared to not receiving day care.

In this paper, we report on this evaluation study which focused on the following questions:

1. Does a broader and larger group of people with dementia and caregivers utilize the broadened offer of Meeting Centers? What are the characteristics of participants (people with dementia and caregivers) in the new interventions? Do they differ from the participants in the regular group-oriented MCSP and people not using day care?

2. What is the effectiveness of the new interventions of the iMCSP compared to the regular MCSP offer on the person with dementia's self-esteem, neuropsychiatric problems, experienced autonomy, and quality of life, and the caregiver's sense of competence and quality of life. And what is the effect of iMCSP, compared to not receiving day care, on people with dementia's and carers' happiness and quality of life?
3. How do people with dementia and caregivers who participate in the iMCSP interventions appreciate the program compared to participants of the regular MCSP?

\section{Method \\ Design}

To answer the first research question, we compared numbers and characteristics of participants of the Meeting Centers that offered iMCSP in addition to the regular MCSP to numbers and characteristics of participants of Meeting Centers that offered only the regular MCSP.

To answer the second research question (effect evaluation) we conducted an explorative randomized controlled trial (randomization at the center level), with two experimental groups and a control group. The Meeting Centers in the experimental groups $(n=16)$ provided the new 'individualized' offer (iMCSP) in addition to the regular MCSP, ie, 11 centers offered DemenTalent, 5 centers offered Dementelcoach + STAR, and 4 centers offered all three interventions. The control group received the regular MCSP $(n=13)$.

In addition, by a pretest-posttest (nonrandomized) control group design, we compared iMCSP with a No day care or caregiver support group (reference group) for which existing data were utilized from the national database "The Older Persons and Informal Caregivers Survey Minimum DataSet" (TOPICS-MDS; This is a public data repository and a questionnaire (one for the older person and one for the informal caregiver) which contains information on background characteristics and the physical and mental health and well-being of older persons and informal caregivers across the Netherlands).

Participants in the new interventions were compared (see "Outcome measures and data collection") with participants in the regular MCSP control group and the "No day care/caregiver support" reference group. Measurements in all groups took place before the start of participation (baseline) and after 6 months. To be able to demonstrate medium effects (power $0.80, \alpha=0.05$, dropout $25 \%$ in 6 months), we aimed at 85 participants per group. ${ }^{24}$

\section{Setting, participants, and randomization procedure}

The study took place in 29 Meeting Centers (16 experimental, 13 control) for people with dementia and their informal caregivers (see Figure 1). Meeting Centers that had been operational for more than 1.5 years were invited 
to join the research by means of a general mailing to all Meeting Centers in the Netherlands and an announcement at the Annual meetings of the National Working Group of Meeting Centers in February 2015 and 2016. The centers that were willing to participate were allocated to the experimental and control interventions by means of the following randomization procedure: the centers were first divided into 4 strata (large/medium cities and north/south regions of the country). After that, 32 meeting centers were randomly drawn from each of these strata by an independent researcher: 16 centers were placed in the experimental group and 16 centers were assigned to the control group. Finally, in the experimental group (again per stratum), the new interventions were randomly assigned to the individual centers: 8 centers would offer DemenTalent, 4 Dementelcoach and STAR e-Learning, and 4 all three interventions. An "oversampling" of centers offering DemenTalent $(n=12)$ was planned, because the implementation of this intervention, as well as the recruitment of participants, was expected to require more time than the informal caregiver interventions (Dementelcoach and STAR). The control groups offered regular MCSP or No day care and caregiver support, respectively. This final control group ( $\mathrm{N}=189$ dyads) was a reference group selected from the already existing national TOPICS-MDS database.

However, 8 centers ( 4 exp, 4 control) dropped out before data collection started ( 6 in the first year of the study and 2 in the second year of the study) because of organizational reasons, and 1 center dropped out after data collection started due to financial reasons. Six of these centers (4 exp, 2 control) were replaced by newly recruited centers, which were again randomized between the experimental and control group (as we were 2 Meeting Centers short, we used 2 dummies during randomization). As 1 center in the control group never collected any data, this finally resulted in 29 participating centers (see Figure 1). Of the 16 centers in the iMCSP group, 7 offered only DemenTalent, 5 Dementelcoach/STAR, and 4 all three interventions (exp. Group), and 13 centers offered the regular MCSP (control group).

New participants in the iMCSP interventions and regular MCSP were invited (orally and in writing) to take part in the research according to an "informed consent" procedure.

\section{The interventions} Experimental group: iMCSP

1) Participants of DemenTalent were deployed, based on their interest, talents, and abilities, as "volunteers" at, among other places, sports associations, green area maintenances, schools, and churches. Interests and talents were investigated by means of individual conversations or group meetings after which the project leader would look for an appropriate workplace in the neighborhood. Guidance was offered by personnel at the workplaces and by personnel of the Meeting Centers (eg, transportation to the workplace, monitoring).

2) Informal caregivers using Dementelcoach received tailored telephone coaching sessions (max. 10).

3) Informal caregivers who registered for the STAR eLearning course received information on how to provide person-centered care to people with dementia and how to take good care of themselves as caregivers by means of 8 training modules.

\section{Control group: regular MCSP}

The regular MCSP consisted of a day club for people with dementia, discussion groups and informative meetings for their caregivers, and a consultation hour, regular center meetings, and social activities for both. In the Meeting Center's day club, people with dementia participate in a variety of recreational and creative activities. Person-centered care is offered with a focus on reactivation, resocialization, and optimization of emotional functioning, depending on experienced problems, needs, wishes and abilities. The day club has a free character; none of the visitors is expected to do anything they do not like. The informative meetings for the caregivers address themes such as the dementia diagnosis, dealing with behavior changes, use of medication, available care and welfare services in the neighborhood, legal and ethical aspects. Some centers organize these meetings together with the nearest Alzheimer café. In the regular center meetings, the voices of all involved in the Meeting Centers (people with dementia, caregivers, and staff) are heard, and suggestions for (new) program activities are discussed.

\section{Implementation of the new "tailored" iMCSP interventions}

To implement the new interventions, the staff of the Meeting Centers who were responsible for the implementation received a two-day training, followed by "coaching on the job" provided by the private company Dirkse Anders Zorgen (DAZ), the Dementelcoach cooperative association, and the Amsterdam Center on Aging of VU University Medical Center (VUmc). 


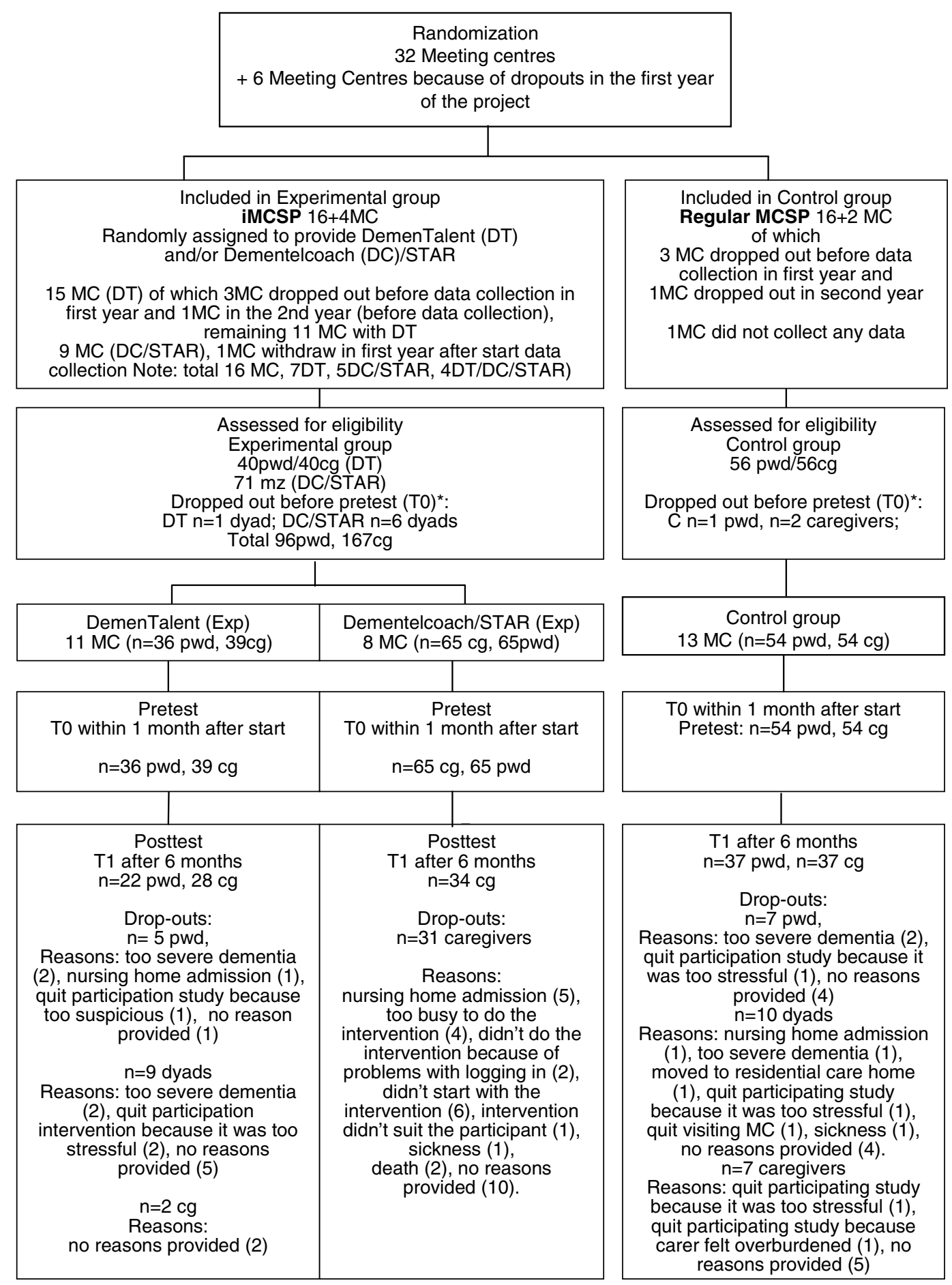

* Reasons for dropout Experimental group: Too severe dementia for DT(1 dyad), caregiver DC/STAR started another intervention (1), DC/STAR no reason provided (5); Control group: sickness pwd (1), caregiver no reason provided (2)

Figure I Flow chart of participants in the iMCSP and MCSP control groups.

Abbreviations: iMCSP, individualized Meeting Centers Support Program; MCSP, Meeting Centers Support Program; DT, DemenTalent; DC/STAR, Dementelcoach/STAR; pwd, person with dementia; cg, caregiver.

For the implementation of DemenTalent, the training consisted of information on the implementation process (eg, How to involve people with dementia in the implementation? How to uncover talents of people with dementia? How to involve workplaces? How to match volunteers to workplaces? Communication plan, 
collaboration network, financing) and information on the content of DemenTalent (eg, looking at abilities of people instead of care needs only, out-of-the-box thinking, change in attitude of professional caregivers, evaluation and monitoring). Furthermore, DAZ provided coaching for the quartermaster activities, such as exploring local partners, workplaces for volunteers, and specific needs and wishes of people with dementia; guiding, supporting, and training of local partners at workplaces/companies who were not familiar with dementia; and finding a professional project leader and a project leader with dementia.

The Dementelcoach cooperative association offered the Meeting Centers guidance on how to inform potential participants and referrers on this new support intervention for caregivers (by means of making local brochures and face-to-face contact and press releases) and explained the procedure of how to refer to the national Dementelcoach office to match participants and coaches.

Information on the content of the STAR e-Learning course was provided to the Meeting Centers by VUmc, which also arranged the course registration of prospective participants and provided a technical helpdesk for support.

In addition, support was provided to all Meeting Centers in the experimental group for the acquisition of (start-up and structural) funding from the municipalities (within the framework of the Social Support Act, WMO) for the implementation of the three new iMCSP interventions.

\section{Outcome measures and procedure of data collection}

For this research, we used quantitative methods.

- Numbers of users of Meeting Centers (research question 1): At the Meeting Centers in the experimental group, the numbers of participants (and attendance) of participating people with dementia and informal caregivers were registered before the introduction of the new interventions (Spring 2016) and at the end of the data collection (Summer 2018). These data were also collected in the control group.

- Data collection effect study (research question 2): For the data collection of the effect study, at two moments ( 0 and 6 months), trained researchers administered standardized (reliable and valid) questionnaires to participants in the experimental group and control group who had given permission to participate in the study. The questionnaires concerned:

- Background characteristics (by TOPICS-MDS questionnaires for older person and caregiver; age, sex, civil status, ethnic background, living situation, SES, education, experienced health, well-being, caregiver burden), diagnosis dementia (according to DSM-5 criteria), severity of dementia by the Global Deterioration Scale), ${ }^{25}$ care needs by the Camberwell Assessment of Needs for the Elderly (CANE), ${ }^{26}$ and activities by the Pleasant Activity List $(\mathrm{PAL})^{27}$ Background data and diagnosis were requested from the family caregiver by telephone (diagnosis if necessary via the general practitioner), the severity of dementia from the program coordinator of the Meeting Center. For the background data of the No day care reference control group, we used existing data from the TOPICS-MDS database.

- People with dementia participating in DemenTalent: (primary outcomes) self-esteem, measured by the Self-esteem scale, ${ }^{28}$ neuropsychiatric symptoms measured by the Neuropsychiatric Inventory (NPI), ${ }^{29}$ (secondary outcomes) experienced autonomy measured by the Experienced autonomy scale, ${ }^{30}$ quality of life measured by the Dementia Quality of Life scale (DQoL), ${ }^{31}$ and QOL-AD, ${ }^{32}$ both recommended by the INTERDEM Group. ${ }^{33}$ The Rosenberg Self-esteem scale is a widely used self-report scale for individual self-esteem consisting of 10 statements (eg, "Overall I am satisfied with myself", "Sometimes I feel useless", "I feel I have good qualities") scored on a 4-point Lickert scale ranging from strongly agree to strongly disagree (score range 10-40). The scale has good internal consistency (alpha $=0,71)$ and test-retest reliability (Pearson's $r=0,85$ ). The NPI-Q consists of 12 items on neuropsychiatric symptoms (such as depression, anxiety, agitation, delusion, apathy) assessing the occurrence (yes/no, range $0-12$ ) and the severity of symptoms (on a 3-point scale), and caregiver distress (scored on a 5-point scale) caused by these symptoms over the past month. The Dutch translation showed sufficient reliability and validity (Kat et al, 2002; de Jonge et al, 2003). ${ }^{34,35}$ The Experienced Autonomy scale is a Dutch scale measuring mastery and selfdetermination. The scale was based on the Mastery scale of Pearlin and the WHOQOL100 and consists of 12 items (eg, "I have no control over things happening to me", "I can decide where I go", "I am able to decide by myself') scored on a 5-point scale (range 12-60). ${ }^{30}$ The scale has not been tested on its psychometric properties yet. The DQoL is a self-report questionnaire (30-items, 5-point visual scale) administered to persons 
with mild-to-moderate dementia to assess their quality of life. It consists of five subscales (score range 1-5) and one overall question on experienced QoL, showing good internal consistency (alpha $=0.67-0.89$ ) and testretest reliability (Pearson's $\mathrm{r}=0.64-0.90)^{31}$ A higher score indicates a better quality of life. The QOL-AD is a 13-item structured interview (scored on a 4-point scale; score range 13-52), which is administered to people with dementia. Interrater and test-retest reliability and internal and criterion validity are good. ${ }^{32,33}$ TOPICS-MDS data (two items on quality of life and well-being/happiness, respectively) were used to compare the DemenTalent (iMCSP) group and MCSP group with the No day care reference group of which existing data from the TOPICS-MDS database were used. Of people with dementia related to caregivers participating in Dementelcoach and STAR, only the NPI and relevant TOPICS-MDS data were collected. Except for the NPI and these TOPICS-MDS data, which were collected by telephone from the caregiver, all questionnaires for the person with dementia were administered face to face by trained researchers.

- The informal caregivers of persons with dementia using DemenTalent and caregivers using Dementelcoach or STAR: (primary outcome measure) Short sense of competence scale ${ }^{34}$ and (secondary) emotional impact of neuropsychiatric symptoms (NPI burden subscale, see above ${ }^{29}$ ) quality of life, and happiness (by two TOPICS-MDS items). The Short Sense of Competence Questionnaire (SSCQ) consists of 7 self-report items, scored on a 5-point Lickert scale from "strongly agree" to "strongly disagree", to assess the family caregiver's feeling of competence. The scale has good internal validity (alpha $=0.76$ ). Construct validity was supported by a high Pearson correlation (0.88) with the original 27-item Sense of Competence Questionnaire. ${ }^{36}$ Trained researchers administered these questionnaires to caregivers by telephone. TOPICS-MDS data (two items on quality of life and wellbeing/happiness) were used to compare the Dementelcoach/STAR e-Learning group with the No day care reference group of which existing data from the TOPICS-MDS database were used.

- Other possible influencing factors: Throughout the research period, the participation frequency was registered for the (different types of) interventions in the experimental and control group, as well as the type of volunteer work at DemenTalent, special events, use of medication, illness around the measurement period, and dropout reasons, such as nursing home admission or death.

- Satisfaction survey (question 3): to investigate the satisfaction of participants with the interventions, they were all invited after 6 months to participate in a survey formulated specifically for this project. ${ }^{37}$ People with dementia were interviewed face to face and caregivers were interviewed by telephone by means of a questionnaire with fixed response alternatives. Questions to the persons with dementia focused on their satisfaction with the activities provided by the interventions, and questions to carers focused on their satisfaction with the information, practical, emotional and social support they received through the interventions.

\section{Analysis}

All statistical analyses were performed using the Statistical Package for the Social Sciences (SPSS) 22.0. The quantitative data concerning characteristics and numbers of participants in the regular Meeting Centers (control group) and the new interventions in the iMCSP (experimental group) were analyzed using descriptive statistics and (non)parametric difference tests ( $\mathrm{t}, \mathrm{Chi}^{2}$, and MannWhitney U tests). Based on the background characteristics, diagnoses, and care needs of participants at baseline, we checked whether the participating Meeting Centers in the experimental and control groups could be treated as homogeneous groups in the analysis. The effect evaluations of the new interventions for people with dementia and for the caregivers were based on an intention-to-treat analysis, including participants with at least one measurement. Multilevel analyses were performed on the outcome measures (M6), taking into account the baseline measurements (M0) and adding potential confounding variables in the analysis. Variables that were added as potential confounders were memory and those variables that differed between the intervention and control group(s) at baseline and were related to the outcome measures. Post-hoc multilevel analyses were performed in which we corrected for potential between-group differences in outcome measures at baseline, including the same potential confounders.

We also checked whether differences in frequency of participation and other variables/factors (special life events, changes in medication use and illness around the time of the measurements, and dropout) had influenced the effect. 


\section{Medical ethical review and trial registration}

The research was assessed by the Medical Ethical Review Committee of the VUmc, which concluded that the study was not subject to the Medical research Involving Human Subjects Act. The trial was registered at the Dutch Trial register (NTR TC 5521).

\section{Results}

\section{Comparison of characteristics iMCSP} and regular MCSP centers (research question I)

At the start of the study, the centers in the iMCSP group $(\mathrm{N}=16)$ and control group $(\mathrm{N}=13)$ did not differ with respect to the time period (mean years) that they were operational or the mean days they were open per week (see Table 1). The total number of participants between the iMCSP and regular Meeting Centers did not differ, but there were more people with dementia/caregiver dyads that participated in the iMCSP group than in the regular Meeting Centers. In the latter, there were more other people that used the support program. After implementation of the new interventions, there was a tendency of more participants utilizing the iMCSP centers $(\mathrm{t}(24)=2.04 ; p=0.053)$. Before and after implementation, there were no significant differences between the iMCSP and regular MCSP centers in number of caregivers that actively used the regular support activities organized specifically for caregivers.

A total of 82 people in 11 Meeting Centers $(M=7.5$ people per Meeting Center; min. 1, max. 16) participated in DemenTalent during the project after the implementation phase (start dates varied between centers from August 2016 to February 2018 and final data on number of participants were collected in December 2018). Onethird of them $(n=27 ; M=2.5$; min. 1, max. 12) participated in the volunteer work exclusively, while two-thirds $(n=55)$ combined DemenTalent with the regular MCSP (after a while).

The mean number of caregivers per Meeting Center ( $n=8$, data of 1 center are missing) that participated in Dementelcoach or STAR e-Learning during the project period and was willing to take part in the research was 2.6 (min. 0, max. 8) and 6.1 (min. 1, max. 14), respectively. It should be noted that there were two centers that did not offer Dementelcoach (due to lack of funding by the community). In the other six Meeting Centers, the mean number of caregivers using Dementelcoach was 3.5.

Table I Characteristics of the iMCSP centers and regular MCSP centers at start of the study

\begin{tabular}{|c|c|c|c|c|}
\hline Characteristics & $\begin{array}{l}\text { iMCSP } \\
\text { M(SD) }\end{array}$ & $\begin{array}{l}\text { Regular } \\
\text { MCSP } \\
\text { M(SD) }\end{array}$ & $t$ Test & $p$ \\
\hline $\begin{array}{l}\text { Number of Meeting Centers } \\
\text { Period Meeting Center is operational at start of study (in years) } \\
\text { Reference date: I-4-20I6 } \\
\text { Days open per week } \\
\text { Before implementation (I-5-20I6) } \\
\text { Number of people with dementia using the Meeting Center } \\
\text { Number of caregivers using the carer support activities (eg, informative } \\
\text { meetings, discussion groups an individual consultation) } \\
\text { Others } \\
\text { After implementation (Nov/Dec 20/8) } \\
\text { Number of people with dementia using regular MCSP } \\
\text { Number of Caregivers using regular MCSP } \\
\text { Others } \\
\text { Mean number of caregivers participating in Dementelcoach (iMCSP) } \\
\text { Mean number of caregivers using STAR e-Learning (iMCSP) }\end{array}$ & $\begin{array}{l}\mathrm{N}=16 \\
8.27(6.56) \\
4.63(1.36) \\
33.80(|8.2|)^{\mathrm{a}} \\
24.93(\mid 5.57)^{\mathrm{a}} \\
1.93(4.08)^{\mathrm{a}} \\
34.94(\mid 7.33) \\
20.06(\mid 3.2 \mathrm{I}) \\
2.50(5.28) \\
(\mathrm{n}=8) \\
3.0 \\
(\min =0, \max =8) \\
(\mathrm{n}=8) \\
6.1 \\
(\min =1, \max =14)\end{array}$ & $\begin{array}{l}\mathrm{N}=13 \\
8.14(5.36) \\
4.38(0.96) \\
19.08(8.28) \\
17.46(15.68) \\
10.00(17.50) \\
24.08(9.88) \\
20.92(15.63) \\
2.00(2.97)\end{array}$ & $\begin{array}{l}t(27)=0.06 \\
t(27)=0.54 \\
t(26)=2.68 \\
t(26)=1.26 \\
t(26)=-1.74 \\
t(27)=2.01 \\
t(27)=-0.16 \\
t(27)=0.30\end{array}$ & $\begin{array}{l}p=0.955 \\
p=0.596 \\
p=0.013 \\
p=0.218 \\
p=0.094 \\
p=0.055 \\
p=0.873 \\
p=0.763\end{array}$ \\
\hline
\end{tabular}

Note: ${ }^{a}$ Data from one Meeting Center are missing.

Abbreviations: MCSP, Meeting Centers Support program; iMCSP, individualized Meeting Centers Support Program. 


\section{Comparison of characteristics}

\section{participants in DemenTalent and regular}

MCSP (research question I)

The participants in DemenTalent that participated in the effect evaluation $(\mathrm{N}=39)$ can be characterized as follows: the average age was 77 , the majority was male (59\%), married or had a partner $(70 \%)$, had a Dutch background $(83 \%)$, had followed vocational training $(31 \%)$, pre-university $(11 \%)$ or university-education $(26 \%)$, did not use home care $(81 \%)$, had Alzheimer's disease $(51 \%)$ or Vascular dementia (17\%), and had mild cognitive decline to mild dementia (94\%; based on GDS).

The participants in DemenTalent matched the participants in the regular MCSP $(\mathrm{N}=54)$ on a number of characteristics, namely SES, ethnicity, experienced health, experienced well-being, total number of care needs (measured with the CANE), performing activities, type of dementia diagnosis, severity of dementia, and comorbidity (see Table 2; detailed information on all background characteristics can be obtained from the researcher on request).

But participants in DemenTalent also differed from the participants of the regular Meeting Centers on certain characteristics, namely they were younger, more often male, less often widow/widower, more often living independently with a partner, higher educated, made less use of home care, and had less unmet needs for daytime activities and company (see Table 2).

Participants in DemenTalent also differed from the reference control group not using day care: they were younger, more often male, married or living with a partner, higher educated, and they experienced better well-being, whereas their caregivers experienced less health and felt more burdened (see Table 2).

\section{Comparison of characteristics of participants in Dementelcoach and STAR and regular MCSP (research question I)}

The participants in the Dementelcoach/Star group ( $\mathrm{N}=65)$ can be characterized as follows (see Table 3): They had an average age of 63 , the majority was female $(62 \%)$, married or long-term living with a partner $(89 \%)$, were husband/wife or partner (51\%) of a person with dementia, had a Dutch ethnic background (90\%), or had completed (pre)university education or higher professional education (53\%).

The caregivers in the Dementelcoach/STAR group matched the caregivers in the regular MCSP $(\mathrm{N}=54)$ on a number of characteristics, namely age, sex, ethnicity, SES, education, experienced health, well-being, objective burden and subjective burden and unmet needs regarding information and psychological distress (see Table 3; detailed information on all background characteristics can be obtained from the researcher on request). Also regarding a number of characteristics of the persons with dementia they cared for (age, sex, ethnicity, SES, home care use, type and severity of dementia), they matched with caregivers using the regular MCSP.

But participants in Dementelcoach/STAR also differed from the participants of the regular Meeting Centers on certain characteristics, namely (see Table 3) they were more often married with or partner of the person with dementia, experienced higher emotional burden of neuropsychiatric symptoms. The persons with dementia they cared for had more severe neuropsychiatric symptoms, higher education, less comorbidity, less unmet needs for daytime activities and company.

Compared to caregivers of the reference control, No day care/respite group caregivers in the Dementelcoach/ STAR group experienced higher subjective burden, and the persons they cared for were more often cohabiting and had higher education (see Table 3).

\section{Effect evaluation of iMCSP - DemenTalent compared to regular MCSP and No day care}

Compared to people with dementia who participated in the regular MCSP, significantly fewer behavioral and mood symptoms (as measured by the Neuropsychiatric Inventory) were observed in participants of DemenTalent after 6 months. Also the severity of the symptoms proved less. Both effects had a moderate effect size $\left(\right.$ eta $^{2}=0.065$ and 0.097 , respectively). We checked if changes in the available data on psychopharmaca use in the DemenTalent and regular MCSP groups explained these outcomes, which was not the case. We also checked if a higher frequency of participation in the DemenTalent group might have influenced these effects. It did not: mean frequency of participation in the DemenTalent group was half a day per week and for the regular MCSP two days per week. Finally, we checked for number and type of life events among people with dementia in the past month, which did not differ significantly between the DemenTalent group and the regular MCSP group ( $\mathrm{Chi}^{2}=0.097, p=0.76$ ). 


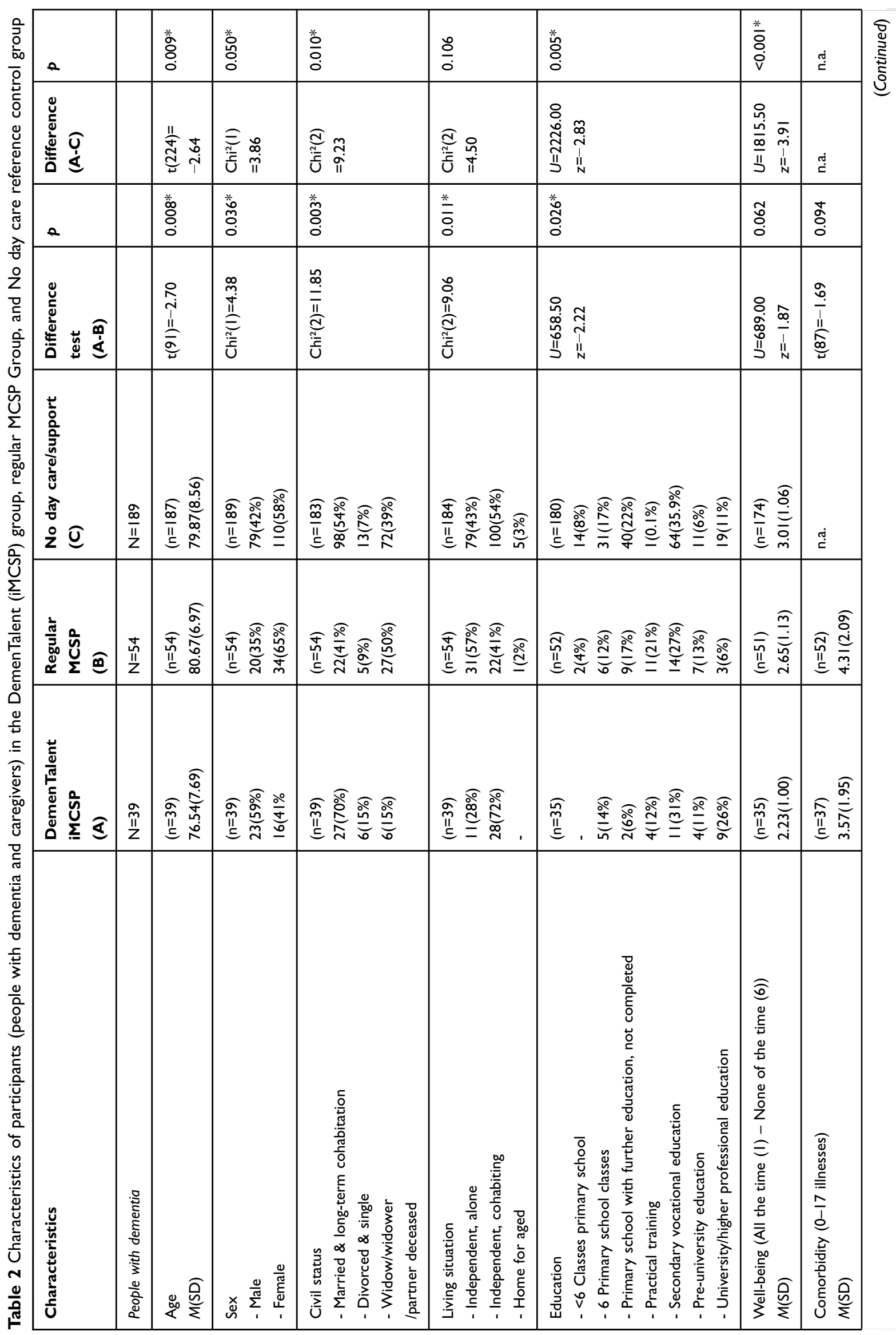




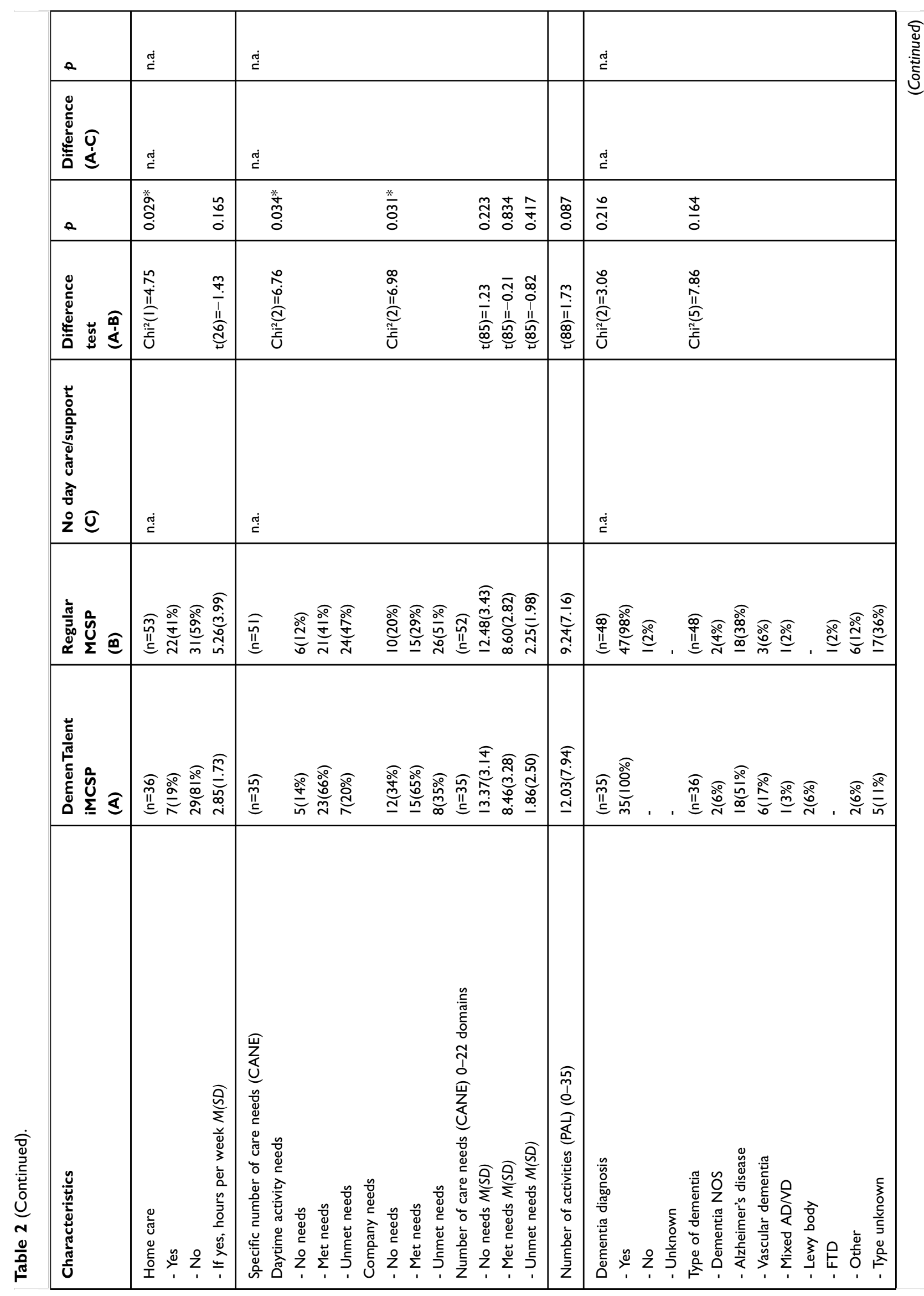




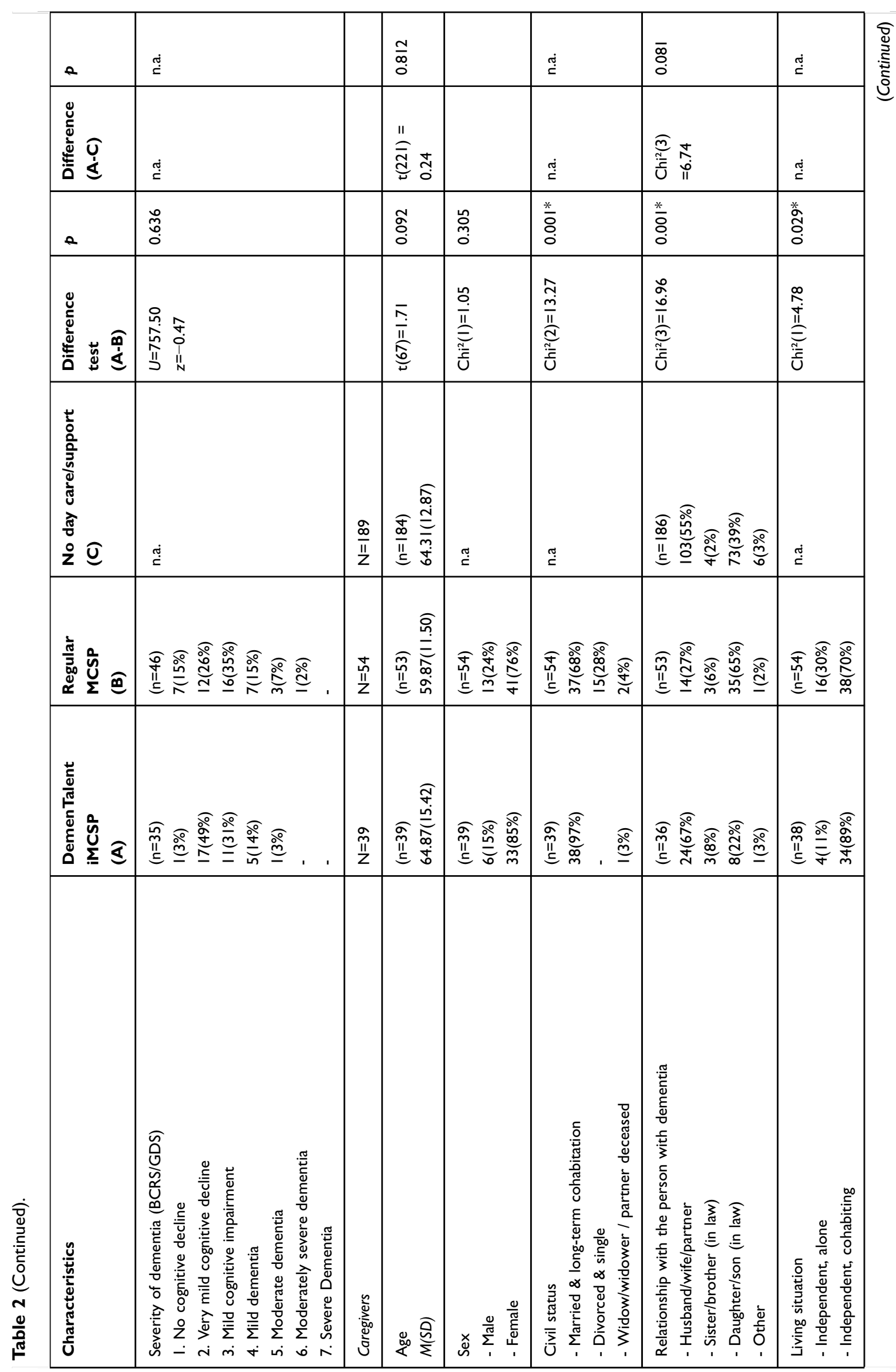




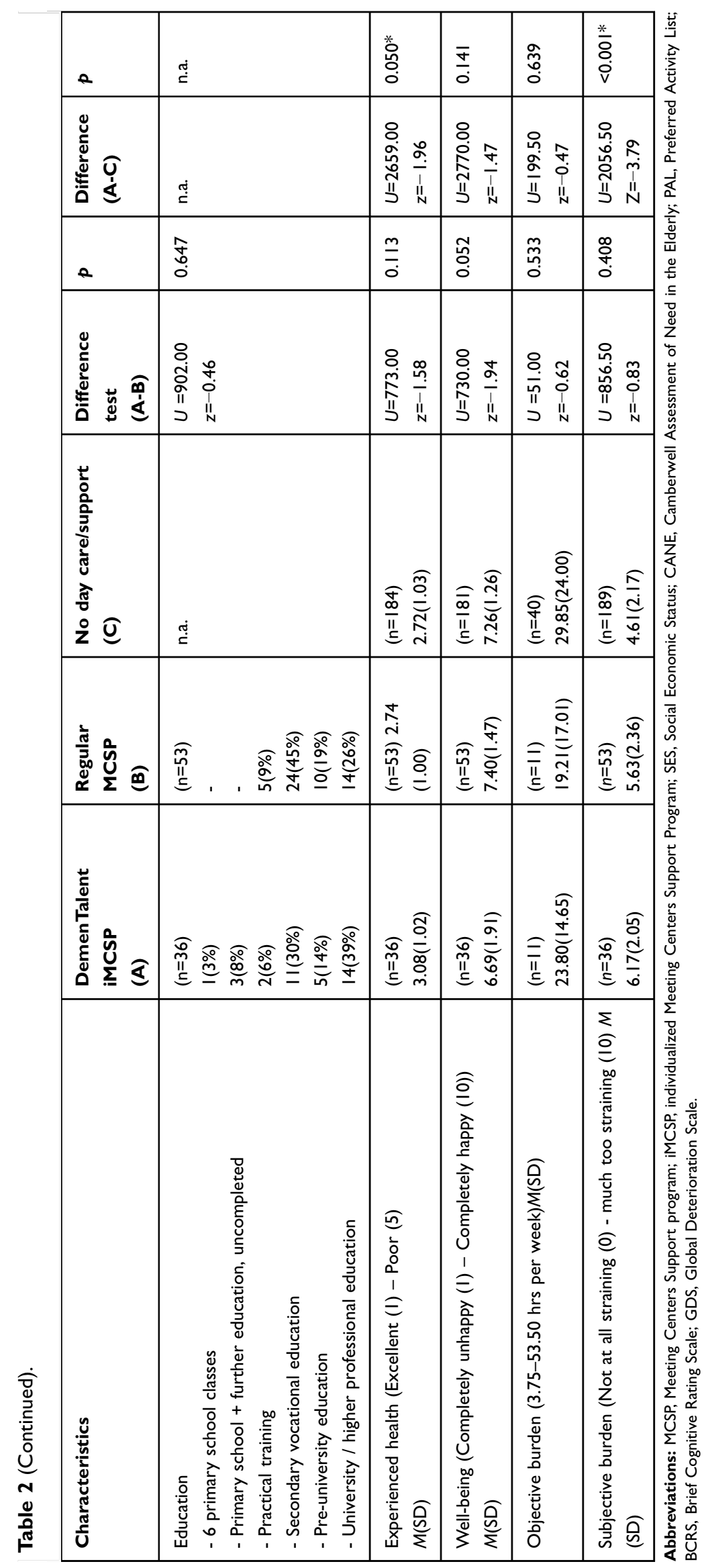




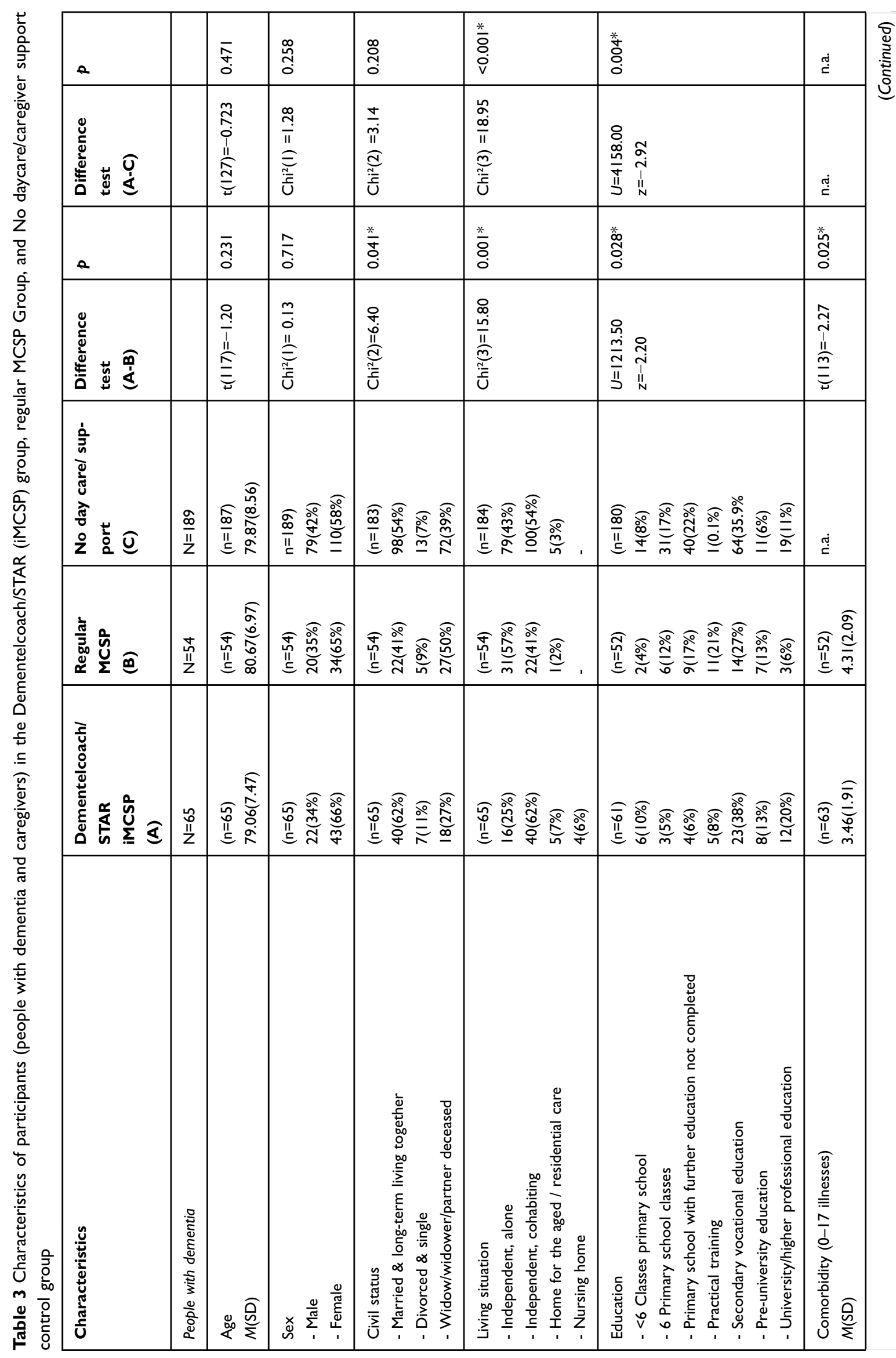




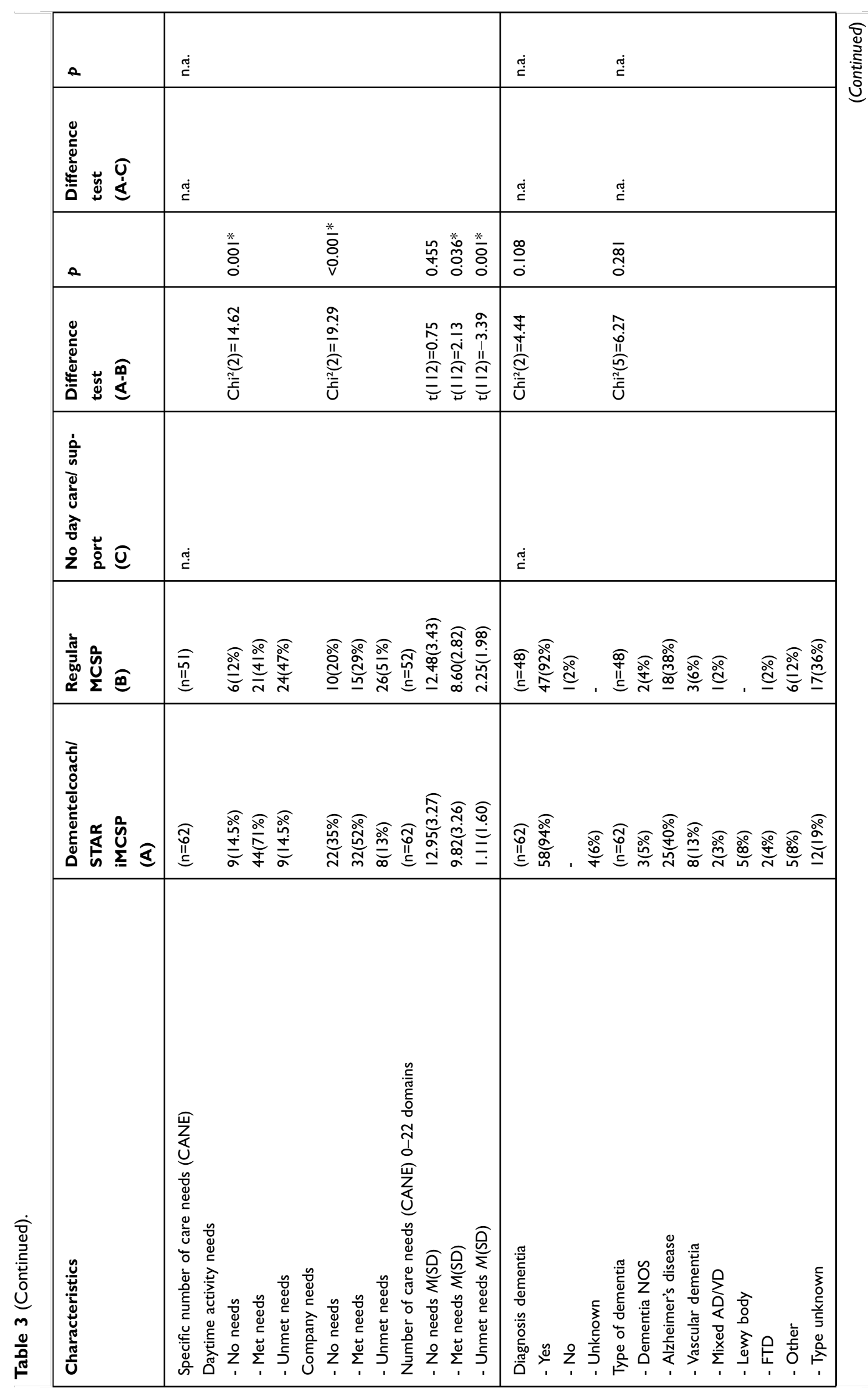




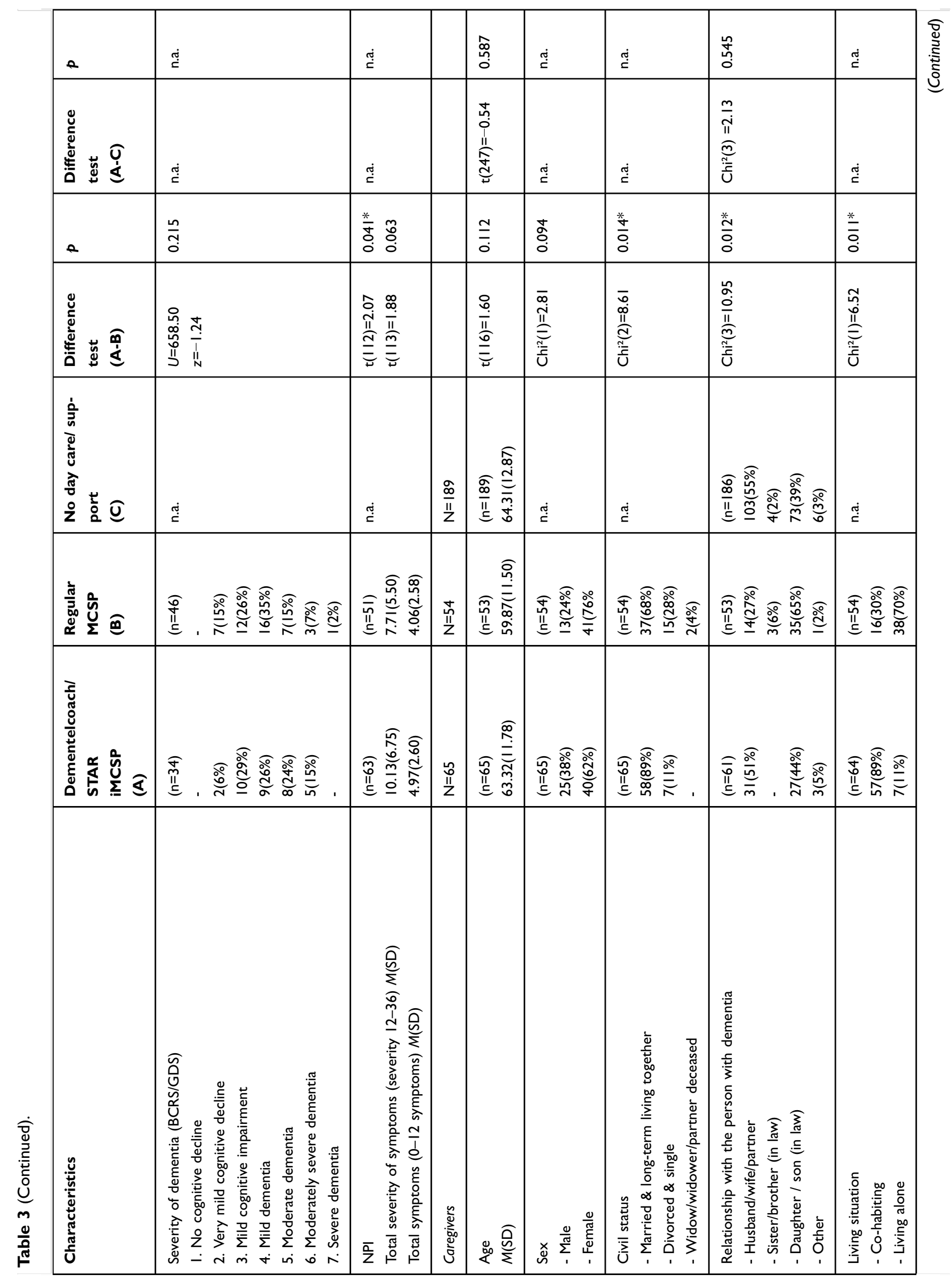




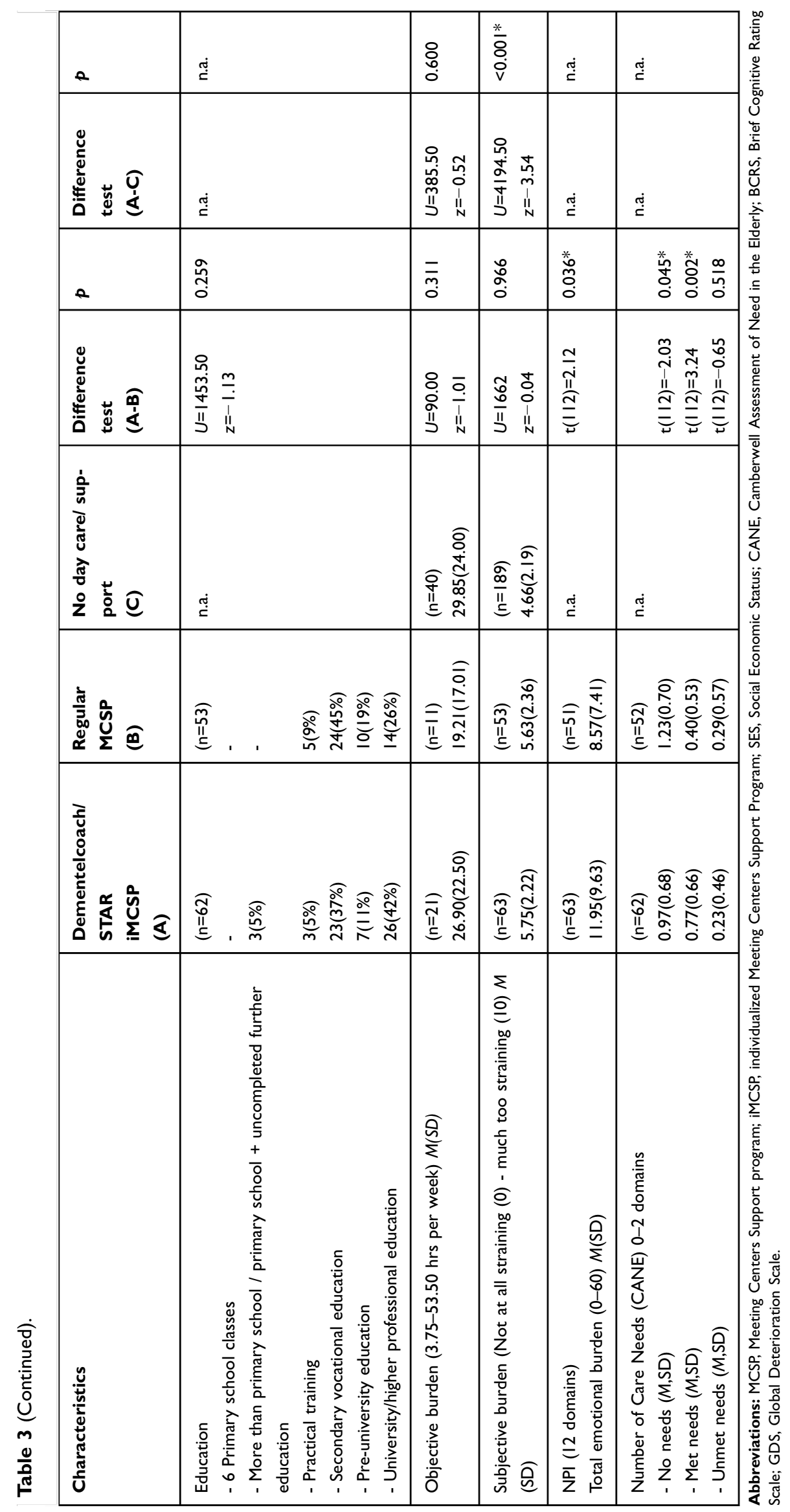


Both in people with dementia who used the regular MCSP and in those who participated in DemenTalent, the MLA showed an increase in the DQoL-positive effect $(\mathrm{B}=0.17, \mathrm{CI}=0.03-0.30 ; p=0.021)$ after 6 months. This time effect, however, did not differ significantly between the groups, although the clinical effect size $\left(\mathrm{eta}^{2}=0.04\right)$ shows a small advantage for the DemenTalent group. No statistical significant differences in effects were observed between the conditions on the other outcome variables: self-esteem, perceived autonomy and other domains of quality of life, although the effect sizes show a small to moderate benefit on negative affect (DQoL; eta $\left.^{2}=0.051\right)$ in favor of the DemenTalent group.

Compared to the reference control group receiving No Day care, no statistical significant differences in QoL $(\mathrm{B}=0.27 ; \mathrm{CI}=-0.09-0.62 ; p=0.139)$, happiness $(\mathrm{B}=0.20 ; \quad \mathrm{CI}=-0.24-0.64 ; p=0.37)$, or feeling sad $(\mathrm{B}=0.28 ; \mathrm{CI}=-0.23-0.78 ; p=0.28)$ were observed in the multilevel analyses (MLA) after six months. Effect sizes (based on ANCOVA completers analyses) were small for all these outcome measures: for QOLGeneral eta ${ }^{2}=0.015$; for Happiness eta ${ }^{2}=0.006$; for Sad eta $^{2}=0.019$.

For the caregivers of people participating in DemenTalent compared to the regular MCSP, the MLA showed a statistically significant effect in experienced burden by neuropsychiatric symptoms after six months in favor of the DemenTalent group (Table 5): they showed a decrease of (-)3.38 whereas in the regular MCSP group there was an increase in experienced burden with 1.24 score (difference 4.62). The ANCOVA on the completers showed a moderate positive clinical effect ( $\mathrm{eta}^{2}=0.06$; see Table 4). No statistically significant differences were found in sense of competence or quality of life after six months.

We checked if a higher frequency of participation of people with dementia in the DemenTalent group might have influenced the effect on caregivers. This was not the case (mean frequency of participation in DemenTalent was half a day per week and for the regular MCSP two days per week). We also checked for number and type of life events among caregivers in the past month, which did not differ significantly between the DemenTalent group and regular MCSP group $\left(\mathrm{Chi}^{2}=0.483, p=0.49\right)$.

Compared to the reference control group receiving No Day care, the MLA showed a significant effect on happiness of caregivers of DemenTalent-participants after six months $\quad(\mathrm{B}=0.613 ; \quad \mathrm{CI}=0.077-1.148 ; \quad p=0.025$; control group declined with -0.303 and exp group increased with 0.310 points). In the analysis, we corrected for the severity of memory problems (TOPICS-MDS) as a potential confounder. Also the ANCOVA on the completers $(\mathrm{nDT}=22$, No day care $=129)$ showed that caregivers of people in the DemenTalent group were significantly more happy $\left(\mathrm{F}(1)=1.378, p=0.242\right.$, eta $\left.{ }^{2}=0.009\right)$. This was a small effect. No effects were found on caregivers' quality of life in the MLA or ANCOVA completers analysis ( $\mathrm{F}(1)$ $=1.151, p=0.285$, eta ${ }^{2}=0.008$ ), corrected for relationship with the person with dementia and memory.

\section{Satisfaction with DemenTalent compared to regular MCSP}

All people with dementia in the DemenTalent group and almost all people in the regular MCSP reported that they liked or very much liked the DemenTalent volunteer work (60\% and 40\%, respectively) and the regular MCSP (29\% and $63 \%$ ). There was no significant difference in the level of satisfaction between the groups $(\mathrm{U}=288, p=0.218)$. The groups gave a mean grade of 8 ( $\mathrm{sd}=0.92$; DemenTalent) and 8.1 ( $\mathrm{sd}=1.12$; regular MCSP) to the programs (on a scale from 1 to 10 ).

Also, all informal caregivers highly appreciated the volunteer work of the person with dementia (50\% being satisfied and 50\% very satisfied) and the regular MCSP ( $40 \%$ being satisfied and $60 \%$ very satisfied). The level of satisfaction between the groups did not differ ( $U=346.50$, $p=0.463$ ). They gave a mean overall grade of 8.3 $(\mathrm{SD}=1.06)$ and $8.4(\mathrm{SD}=0.74)$ to the DemenTalent voluntary work and the regular MCSP, respectively.

\section{Effect evaluation of iMCSP - Dementelcoach or STAR - compared to regular MCSP and no caregiver support}

For caregivers no significant group $\mathrm{x}$ time effects of Dementelcoach/STAR versus regular MCSP were found regarding experienced burden by neuropsychiatric symptoms, sense of competence, quality of life and happiness, nor regarding quality of life and happiness for Dementelcoach/STAR versus regular MCSP (see Tables 6 and 7). However, the effect sizes for experienced burden, sense of competence, and quality of life show small positive clinical benefits in favor of the Dementelcoach/STAR group (see Table 6).

We checked the actual use of the Dementelcoach and STAR interventions: the mean number of coaching sessions caregivers received was 8.2 (min. 3, max. 11 


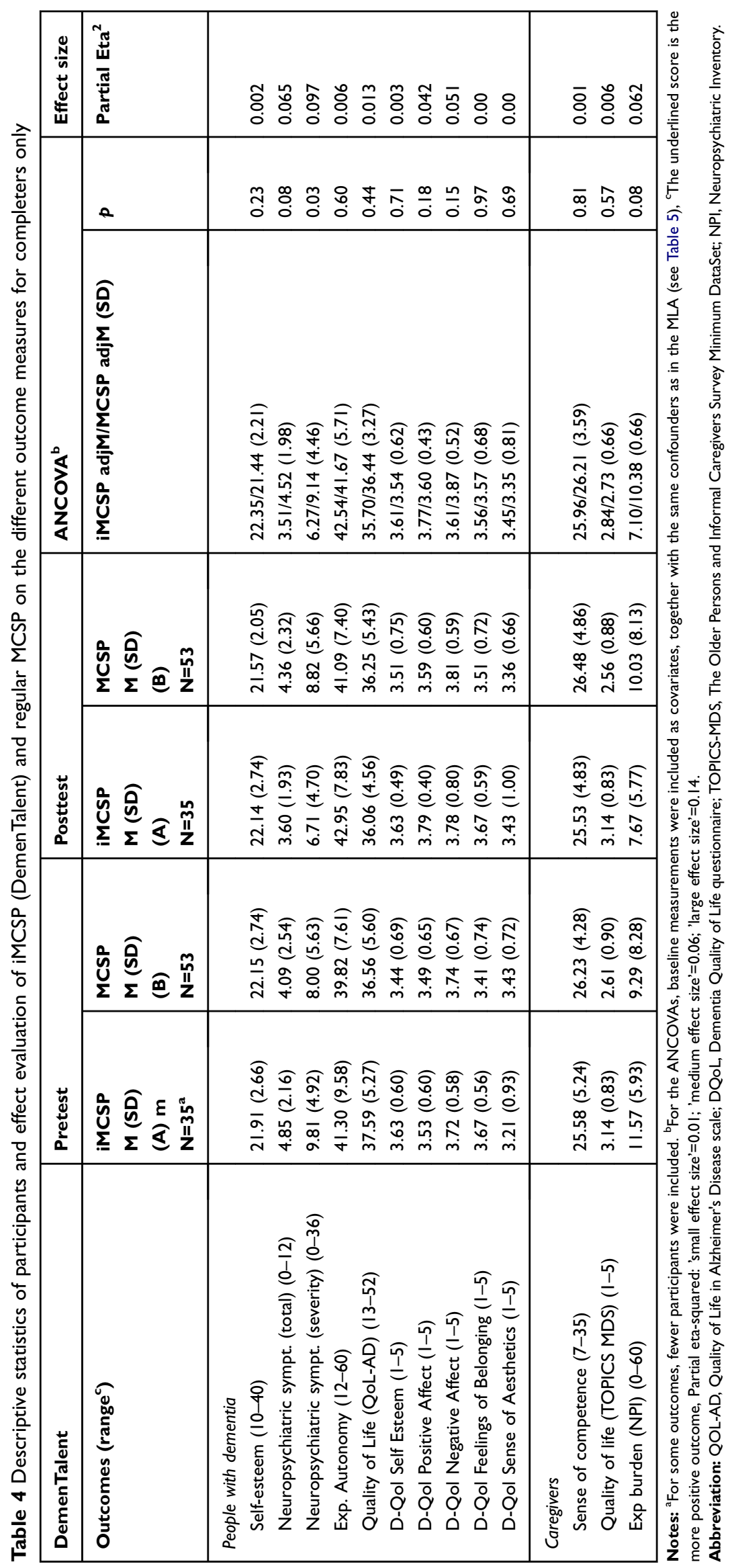


Table 5 Associations between time, group (DemenTalent/Control) and interactions and I 3 outcomes; based on bivariate (unadjusted) linear mixed models

\begin{tabular}{|c|c|c|c|c|}
\hline Outcome variables & $\begin{array}{l}\text { Intercept (mean at t0) } \\
\text { B }\end{array}$ & $\begin{array}{l}\text { Group (ref = control- } \\
\text { MCSP) } \\
\text { B }\end{array}$ & $\begin{array}{l}\text { Time: }(\text { ref = baseline }) \\
\text { B }\end{array}$ & $\begin{array}{l}\text { Time * Group } \\
\text { B }\end{array}$ \\
\hline \multicolumn{5}{|l|}{ People with dementia } \\
\hline Self-esteem $(10-40)^{a}$ & 20.26 & -0.34 & -0.25 & 0.59 \\
\hline NPI tot symptoms $(0-12)$ & 3.59 & 0.82 & 0.21 & $-1.30 *$ \\
\hline NPI symptoms severity $(0-36)$ & 6.59 & $2.50 * *$ & 0.87 & $-3.73 * *$ \\
\hline Exp autonomy $(12-60)$ & 38.36 & 2.09 & 1.03 & -1.20 \\
\hline QoL-AD (I3-52) & 33.57 & 1.25 & -0.27 & -1.28 \\
\hline DQoL self-Esteem $(\mathrm{I}-5)^{\mathrm{b}}$ & 3.05 & 0.23 & 0.07 & -0.05 \\
\hline DQoL positive effect $(I-5)$ & 3.44 & 0.14 & 0.14 & 0.08 \\
\hline DQoL negative effect $(I-5)^{b}$ & 3.84 & -0.10 & 0.11 & -0.13 \\
\hline DQoL feelings of belonging $(\mathrm{I}-5)^{\mathrm{c}}$ & 1.72 & 0.20 & 0.08 & -0.11 \\
\hline DQoL sense of aesthetics $(I-5)$ & 3.60 & -0.03 & 0.04 & 0.14 \\
\hline \multicolumn{5}{|l|}{ Caregivers } \\
\hline Sense of competence $(7-35)^{d}$ & 22.23 & -1.70 & -0.02 & 0.20 \\
\hline Quality of life TOPICS MDS $(I-5)^{e}$ & 2.72 & 0.30 & -0.45 & 0.022 \\
\hline Exp burden (NPI) $(0-60)^{f}$ & 19.35 & 2.06 & 1.24 & $-4.62 *$ \\
\hline
\end{tabular}

Notes: 'With confounding variables: memory, civil status, and education; 'With memory and education; 'With memory and civil status; all other person with dementia' outcomes only with memory as confounder; 'With caregiver QoL and Memory of PwD as confounders; ${ }^{e}$ With caregiver age, Relationship with Pwd and memory of PwD as confounders, ,With caregiver QoL and Memory of PwD as confounders. $* P<0.05 ; * * P<0.01$.

Abbreviation: NPI, Neuropsychiatric Inventory; QOL-AD, Quality of Life in Alzheimer's Disease scale; DQoL, Dementia Quality of Life questionnaire; TOPICS-MDS, The Older Persons and Informal Caregivers Survey Minimum Dataset.

sessions) of 45 mins each; the percentage of people who completed the different STAR modules (these data were available for $39 \%$ of the STAR e-Learning users): Modules 1, 2, and 3 about the diagnosis and living with dementia were most frequently completed $(94 \%, 82 \%$, and $82 \%$, respectively), modules 4 and 6 about practical difficulties in daily life and support strategies by $65 \%$ of the caregivers and modules 5 (emotional impact on behavior and mood of people with dementia) and 7 (positive and empathic communication) by $59 \%$ of the caregivers. A little less than half of the caregivers (47\%) completed the final module 8 (Emotional impact and looking after yourself as a caregiver). We also checked for possible differences in number and type of life events of caregivers and people with dementia in the Dementelcoach/STAR group and the regular MCSP in the past month and found none $\left(\mathrm{Chi}^{2}=2.47, p=0.12 ; \mathrm{Chi}^{2}=0.261, p=0.61\right.$, respectively $)$.

Compared to the national reference control group receiving No Day care/respite the MLA showed a significant positive effect on experienced happiness in the Dementelcoach/STAR group after 6 months $(\mathrm{B}=0.507$; CI $0.068-0.945 ; p=0.024$ ). The effect size (based on ANCOVA completers analyses DC/STAR $n=31$ and No Day care $n=129$ ) was small (eta2 $=0.017$ ).
No effects regarding the quality of life were found in the caregivers receiving Dementelcoach or STAR compared to the reference control group receiving No Day care/respite.

\section{Satisfaction of caregivers using Dementelcoach or STAR compared to regular MCSP}

As high as $37 \%$ of the caregivers of the Dementelcoach (48\%) and STAR group (32\%) filled in the satisfaction survey and $67 \%$ of the regular MCSP group. The large majority proved satisfied or very satisfied with the coaching $(40 \%, 40 \%)$ and the STAR e-Learning course $(64 \%$, $29 \%$ ). One person was unsatisfied and one moderately satisfied about the coaching and one person was moderately satisfied with the STAR e-Learning. The satisfaction on Dementelcoach and STAR e-Learning did not differ significantly from the satisfaction of caregivers on the regular MCSP as a whole $(61 \%, 39 \%$; $\mathrm{U}=160, p=0.541$; $\mathrm{U}=215, p=0.345$ ) or the discussion group (compared to Dementelcoach; $\mathrm{U}=24, p=0.892$ ) and the informative meetings of the regular MCSP (compared to STAR eLearning; $\mathrm{U}=93, p=0.778$ ). 


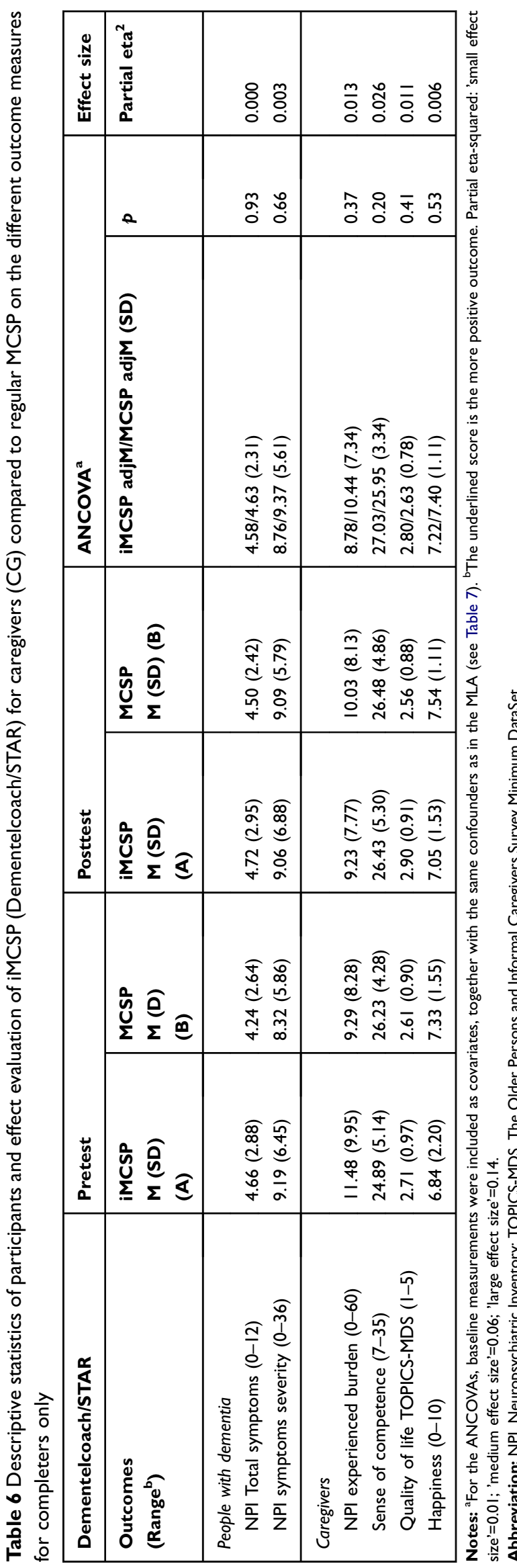

The groups gave a mean grade of $7.8(\mathrm{SD}=1.48)$ for Dementelcoach, $7.8(\mathrm{SD}=0.98)$ for STAR e-Learning, and $8.4(\mathrm{SD}=0.73)$ for regular MCSP (on a scale from 1 to 10$)$.

Of the caregivers who received coaching the large majority reported to have received some $(50 \%)$ or a lot $(40 \%)$ of emotional support. This was comparable to the emotional support experienced by caregivers who participated in the regular MCSP. However, compared to caregivers in the regular MCSP, 79\% of whom said they always receive new information and $60 \%$ a lot of practical support, smaller percentages in the Dementelcoach group reported to have received new information ( $20 \%$ sometimes, $20 \%$ always) or practical support ( $10 \%$ some, $30 \%$ a lot).

As high as $79 \%$ of the caregivers who followed the STAR e-Learning course reported to have received a lot of new information and $21 \%$ some new information. This was much more than in the regular MCSP group $(\mathrm{U}=31.50, p<0.001)$ of which $47 \%$ said to have received a lot and $33 \%$ some new information. There were no significant differences between the STAR e-Learning and regular MCSP group regarding the amount of practical advice caregivers received.

\section{Post-hoc effect analyses}

The post-hoc MLAs with corrections for potential between-group differences in outcome measures at baseline, showed comparable trends for all statistically significant effects described above. Results on the severity of neuropsychiatric symptoms and the emotional impact of these symptoms experienced by caregivers were again statistically significant in favor of DemenTalent $(\mathrm{B}=-2.73 ; \mathrm{p}=0.03$ and $\mathrm{B}=-3.89 ; \mathrm{p}=0.02$, respectively). For the total number of neuropsychiatric symptoms of DemenTalent participants (compared to the regular MCSP group) and for happiness of the caregivers in the DemenTalent and Dementelcoach/STAR group (compared to the caregivers in the No day care group) the results pointed in the same favorable direction, but were no longer statistically significant $(B=-0.93 ; p=0.11 ; B=0.44 ; p=0.07$, and $\mathrm{B}=0.37 ; \mathrm{p}=0.08$, respectively).

\section{Discussion}

The study showed that after implementation of the new iMCSP interventions (DemenTalent, Dementelcoach, and STAR e-Learning) a broader group of participants utilized the broadened support offer of the Meeting Centers compared to the group participating in the regular MCSP. The DemenTalent participants proved younger, more often 
Table 7 Associations between time, group (Dementelcoach/STAR - Control) and interactions and 4 outcomes; Based on bivariate (unadjusted) linear mixed models

\begin{tabular}{|l|l|l|l|l|}
\hline Outcome variables & $\begin{array}{l}\text { Intercept (mean at } \\
\text { t0) } \\
\text { B }\end{array}$ & $\begin{array}{l}\text { Group (ref = control- } \\
\text { MCSP) } \\
\text { B }\end{array}$ & $\begin{array}{l}\text { Time: (ref = base- } \\
\text { line) } \\
\text { B }\end{array}$ & $\begin{array}{l}\text { Time * } \\
\text { Group } \\
\text { B }\end{array}$ \\
\hline $\begin{array}{l}\text { Caregiver } \\
\text { NPI experienced burden (0-60) }\end{array}$ & -2.96 & 2.85 & \\
Sense of competence (7-35) & 25.94 & -0.29 & 0.98 \\
Quality of life (TOPICS-MDS) & 2.21 & 0.19 & -0.18 & -3.43 \\
$(I-5)^{\mathrm{b}}$ & & -0.35 & 0.05 & 0.05 \\
Happiness (0-10) & 5.77 & -0.35 & 0.02 \\
\hline
\end{tabular}

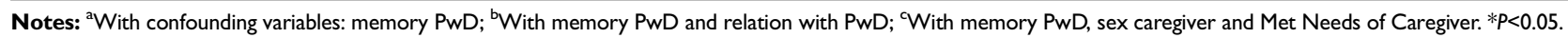
Abbreviation: MCSP, Meeting Centers Support Program; NPI, Neuropsychiatric Inventory; TOPICS-MDS, The Older Persons and Informal Caregivers Survey Minimum DataSet.

male, more often living with a spouse/partner, and less often widowed, they were more highly educated, made less use of home care, and had fewer unmet needs for daytime activities and company. The caregivers using Dementelcoach and/or STAR were more often married with or partner of the person with dementia, experienced higher emotional burden of neuropsychiatric symptoms at the start of the intervention, but had less unmet needs regarding information and psychological distress at the start than caregivers using the regular MCSP.

\section{Effectiveness DemenTalent compared to regular MCSP}

Compared with the regular MCSP for people with dementia, DemenTalent had a moderate positive effect on behavioral and mood symptoms (neuropsychiatric symptoms) and the symptoms also proved less severe. The positive effect of participants (one of the domains of quality of life) had improved both within the DemenTalent group and the regular MCSP group after six months of participation, but no differences were found between the groups. Regarding the other outcomes (self-esteem, experienced autonomy, and other domains of quality of life) in people with dementia, we also found no differences between the groups. However, effect sizes showed that there were small to moderate clinical benefits for positive effect and negative effect in favor of the DemenTalent group. In caregivers of people with dementia who participated in DemenTalent, a significantly moderate positive effect was found on experienced burden due to the neuropsychiatric symptoms of the person with dementia. No differences were found in sense of competence, quality of life, or feeling happy compared to those participating in regular MCSP.
Compared to those receiving No Day care at all, the caregivers of participants of DemenTalent proved happier. No differences were found regarding carers' quality of life.

People with dementia as well as carers were highly satisfied with both the individualized and the regular MCSP.

\section{Effectiveness Dementelcoach/STAR compared to regular MCSP}

Although for caregivers, no significant differences in effects of Dementelcoach/STAR versus regular MCSP were found on the primary and secondary outcomes, the effect sizes for experienced burden, sense of competence, and quality of life showed small positive clinical benefits in favor of the Dementelcoach/STAR group. Compared to the reference control group receiving No Day care/respite the Dementelcoach/STAR group proved a little happier, although their overall quality of life did not differ.

\section{Post-hoc Analyses}

In the post-hoc multilevel analyses, which we conducted for the evaluation of the effectiveness of the iMCSP interventions and in which we corrected for between-group differences on the outcome measures at baseline, we found comparable significant effects for DemenTalent in the same direction for two outcomes (i.e. for severity of neuropsychiatric symptoms and emotional impact of these symptoms on caregivers). For three outcomes (i.e. total number of neuropsychiatric symptoms in the DemenTalent group and happiness of caregivers in all intervention groups) we found nearly statistically significant effects in the same direction, based on an alpha of 0.05 .

Our study shows the benefit of offering people with dementia personalized activities that match their preference, abilities, and talents and helps them fulfill their potential in society. The positive results of DemenTalent 
on neuropsychiatric symptoms are in line with previous studies into person-centered psychosocial interventions that showed positive effects on behavior and mood of people with dementia: A recent comprehensive review by Testad et $\mathrm{al}^{38}$ which included 40 intervention studies conducted in care homes and nursing homes, showed that there is a growing body of evidence indicating specific effects of different personalized psychosocial interventions on individual behavioral and mood symptoms in dementia. Specifically for personalized pleasant activities (with and without social interaction), there was good evidence that supported its value for the treatment of agitation. The findings are also in line with another recent review by Scales et al, ${ }^{39}$ who in addition mentioned physical exercise as one of those pleasant activities that decrease agitation and depression in people with dementia. The majority of the intervention studies have so far been conducted in residential care settings with people with more advanced dementia. There is still relatively little evidence on the effect of personalized psychosocial interventions aiming at fulfilling one's potential in society and social participation on behavior and mood of community-dwelling people with mild to moderate dementia and on their informal caregivers. Good examples are the studies by Gitlin et $\mathrm{al}^{40}$ and Graff et $\mathrm{al}^{41}$ into home-based occupational therapy, the study by Clare et $\mathrm{al}^{42}$ into goal-oriented cognitive rehabilitation; the studies by Dröes et $\mathrm{al}^{10-13}$ and Van Haeften-VanDijk et $\mathrm{al}^{43}$ into the Meeting Centers Support Program and Easy Access Day care centers plus Carer Support which aim to promote social participation and are both based on the adaptation-coping model, ${ }^{44}$ the study by Park ${ }^{45}$ in which people with dementia acted as volunteers, eg, in educational activities for young people, studies into green care farms, ${ }^{46}$ and museum visit programs. ${ }^{47,48}$ The present study into iMCSP, more specifically DemenTalent, adds to the growing body of evidence and insight that it is crucial to be responsive to the perspective of the person with dementia, to support their sense of self and promote individualization and relationship building in an environment that promotes wellbeing. 49

The fact that caregivers in both the DemenTalent group and the Dementelcoach/STAR group were happier than caregivers of people with dementia who did not receive support confirms the findings of a comprehensive meta-review by Gilhooly et $\mathrm{al}^{50}$ including, amongst other reviews, 22 reviews of caregiver intervention studies. They found that psychosocial interventions for the person with dementia and psycho-educational interventions for the caregivers were beneficial for the psychological well-being of caregivers. The increased happiness in caregivers using Dementelcoach or STAR e-Learning in our study is also in line with the study outcome of Steffen and Gant $^{23}$ who demonstrated a decrease in depression among caregivers of people with dementia after telephone coaching.

\section{Limitations of the study}

This study had several limitations. First of all, the sample recruited for the DemenTalent group was smaller than originally planned based on the power analysis we conducted, and there were relatively many dropouts during the study period of six months for different reasons (see Figure 1). As a consequence, the study sample was relatively small. The small-to-moderate effect sizes found for experienced autonomy, self-esteem, positive and negative effect, and caregiver burden in the completers analyses in favor of the DemenTalent group indicate that a larger group will be needed to show statistically significant differences. The same applies to the Dementelcoach/STAR group, for which the effect evaluation showed a positive trend for sense of competence.

Second, not all new participants of DemenTalent and/ or their caregivers and participants of Dementelcoach/ STAR were willing to participate in the evaluation study. As we have no information about the background characteristics and the percentage of people who did not participate in the evaluation study, we cannot check if this has led to any selection bias.

Third, except for happiness and quality of life, no data on the other primary and secondary outcome measures were available for the reference control group who did not use any kind of day care or caregiver support (from the TOPICS-MDS Database). This limited the effect evaluation of most of the outcome measures for DemenTalent to the added value of the volunteer work compared to the regular MCSP. Consequently, based on this study, no general conclusions can be drawn about the effect of DemenTalent on these outcome measures compared to not receiving any day care support. The same goes for Dementelcoach and STAR e-Learning based on our study. However, in previous research, the latter two interventions showed positive effects on caregivers' sense of competence compared to caregivers not receiving these interventions. $^{19,21}$ 


\section{Scientific and clinical value of the study and recommendations}

This is one of the first studies, as far as we know, that focused on the effect of volunteer work for people with dementia that aims to enable them to fulfill their potential in society and to socially participate by providing them the support needed to achieve this goal. The results are promising. Further research in larger samples is recommended to investigate how conducting different types of volunteer work that match their talents influences the feelings of autonomy, self-esteem, and quality of life of people with dementia. Also, further development of methods of personalized goal attainment is necessary to better understand what is important to the person with dementia and their caregivers and to evaluate if goals are achieved, which is particularly important to guide this matching process and to evaluate the outcomes (see also Jennings et al). ${ }^{51}$

The positive results on behavior and mood and the high appreciation of the talent-based volunteer work are sufficient reason to further disseminate and implement DemenTalent and to recommend it to potential users. The beneficial effect of the three iMCSP interventions, DemenTalent, Dementelcoach, and STAR e-Learning on caregivers' feelings of happiness also justify recommending caregivers to use this individualized support offered by the Meeting Centers. In two previous papers ${ }^{22,52}$ we reported on the results of our studies into the implementation of the three new interventions of iMCSP and a practical guide was developed to support Meeting Centers to set up this individualized program. Together with the results of this evaluation study, this will hopefully encourage other centers to adopt iMCSP, which will enable many more people with dementia and caregivers to benefit from it.

\section{Conclusion}

Based on the results of this explorative effect study, we can conclude that the highly appreciated individualized MCSP can be effectively applied as an alternative or additional support program to the regular MCSP for a subgroup of people with dementia and carers who prefer individualized activities and support outside the Meeting Center. DemenTalent proved to decrease the severity of neuropsychiatric symptoms of people with dementia and emotional burden of caregivers. All iMCSP interventions tended to result in caregivers being happier compared to those receiving no support. Large-scale studies are needed to investigate the effect of iMCSP on other domains of quality of life of participants.

\section{Data sharing statement}

- The authors intend to share deidentified data via the TOPICS-MDS Database (public data repository in the Netherlands).

- These data concern data on care receivers and caregivers regarding background characteristics, quality of life, well-being, health; on the care receiver regarding comorbidity, ADL, and care use; on the caregiver regarding objective and subjective burden.

- There were two additional papers accepted on the implementation of iMCSP in Aging and Mental Health (on the implementation of Dementelcoach and STAR e-Learning) and International Psychogeriatrics (on the implementation of DemenTalent), both by Van Rijn, A. et al (2019). There has also been developed a practical guide (Van Dijk, M. \& Dröes, R.M. Draaiboek Ontmoetingscentra3.0; in Dutch, VUmc, Amsterdam, 2019) for Meeting Centers who intend to implement iMCSP at their own center. The trial has been registered in the Dutch Trial register (NTR TC 5521).

- The deidentified data on care receivers and caregivers will be accessible on request via the TOPICS-MDS public data repository https://topics-mds.eu/contact-us/ (e-mail: topics-mds@radboudumc.nl)

- TOPICS-MDS is a public repository with no end date, the data will therefore be available on request as long as there will be funding for this repository.

\section{Ethics approval and informed consent}

The research was assessed by the Medical Ethical Review Committee of the VU University medical center, which concluded that the study was not subject to the Medical research Involving Human Subjects Act. New participants in the iMCSP interventions and regular MCSP were invited (orally and in writing) to take part in the research according to an "informed consent" procedure. The trial was registered at the Dutch Trial register (NTR TC 5521).

\section{Acknowledgments}

This study was funded by the ZonMw Memorabel program, Alzheimer Nederland, Foundation Dijkverzwaring, Foundation Dioraphte, Foundation RCOAK, Foundation Sluyterman Van Loo, Bavo foundation, Hofje Codde en Van Beresteyn Foundation, the Municipality of Amsterdam and Foundation for Support VCVGZ. We would like to thank the people with dementia, informal 
caregivers and personnel of the Meeting Centers who participated in this study. We also thank the members of the sounding board group of this project. Finally, we would like to thank Ruud Dirkse (DAZ) and Nicole de Koning (Dementelcoach) who were part of the Steering group of this project and coached the implementation of the new interventions DemenTalent and Dementelcoach in the participating Meeting Centers, and Joke Bos (Stichting Combiwel Buurtwerk), Anita Schimmel (King Arthur group), Julie Meerveld (Alzheimer Nederland), Saskia Danen (Dementelcoach) and Martin Sturkop (informal caregiver) for their valuable contribution as project advisors throughout the study. The funding organizations had no influence on the data collection, data analyses or reporting. The authors have not entered into an agreement with the funding organizations that has limited their ability to complete the research as planned and publish the results. The authors have had full control of all the primary data.

\section{Author contributions}

All authors contributed to data analyses, drafting or revising the article, gave final approval of the version to be published, and agree to be accountable for all aspects of the work.

\section{Disclosure}

The authors report no conflicts of interest in this work.

\section{References}

1. Alzheimer Nederland. Handreiking (dag)activiteiten Bij Dementie. [guideline (daytime) Activities in Dementia]. Alzheimer Nederland: Amersfoort; 2014

2. Van der Lee J, Bakker TJEM, Van Duivenvoorde H, Dröes RM Multivariate models of subjective caregiver burden in dementia; a systematic review. Ageing Res Rev. 2014;15:76-93. doi:10.1016/j.arr.2014.03.003

3. Cheng S-T. Dementia caregiver burden: a research update and critical analysis. Curr Psychiatry Rep. 2017;19(9):64. doi:10.1007/s11920-0170818-2

4. Van der Heide I, Van Den Buuse S, Francke AL. Dementiemonitor Mantelzorg 2018; Mantelzorgers over Ondersteuning, Zorg, Belasting En De Impact Van Mantelzorg Op Hun Leven. [dementia Monitor Informal Care 2018; Informal Carers on Support, Care, Burden and the Impact of Providing Informal Care on Their Life]. Utrecht: Nivel; 2018.

5. Alzheimer Society UK. Dementia 2013: the hidden voice of loneliness. Alzheimer Society UK. 2013, April. Available from: https://www.alzheimers.org.uk/sites/default/files/migrate/downloads/ dementia_2013 the_hidden_voice_of_loneliness.pdf. Accessed 15 February, 2019.

6. Von Kutzleben M, Schmid W, Halek M, Holle B, Bartholomeyczik S. Community-dwelling persons with dementia: what do they need? What do they demand? What do they do? A systematic review on the subjective experiences of persons with dementia. Aging Ment Health. 2012;16 (3):378-390. doi:10.1080/13607863.2011.614594
7. Van der Roest HG, Meiland FJM, Comijs HC, et al. What do community dwelling people with dementia need? A survey among those who are known by care and welfare services. Int Psychogeriatr. 2009;21(5):949-965. doi:10.1017/S1041610209990147

8. Dröes RM, Boelens-Van der Knoop ECC, Bos J, et al. Quality of life in dementia in perspective; an explorative study of variations in opinions among people with dementia and their professional caregivers, and in literature. Dementia. 2006;5(4):533-558. doi:10.1177/ 1471301206069929

9. Mak S. Naar een betere dagbehandeling; Knelpunten in de dagbehandeling voor mensen met dementie. [Towards better day treatment; Bottlenecks in day treatment for people with dementia]. Denkbeeld. 2010;22(3):6-8. doi:10.1007/s12428-010-0029-7

10. Dröes RM, Breebaart E, Van Tilburg W, Mellenbergh GJ. The effect of integrated family support versus day care only on behavior and mood of patients with dementia. Int Psychogeriatr. 2000;12(1):99116.

11. Dröes RM, Breebaart E, Meiland FJM, Van Tilburg W, Mellenbergh GJ. Effect of meeting centres support programme on feeling of competence of family caregivers and delay of institutionalization of people with dementia. Aging Ment Health. 2004a;8(3):201-211. doi:10.1080/13607860410001669732

12. Dröes RM, Meiland FJM, Schmitz M, Van Tilburg W. Effect of combined support for people with dementia and carers versus regular day care on behaviour and mood of persons with dementia: results from a multi-centre implementation study. Int J Geriatr Psychiatry. 2004b;19(7):1-12. doi:10.1002/gps.1142

13. Dröes RM, Meiland FJM, Schmitz M, Van Tilburg W. An evaluation of the meeting centres support programme among persons with dementia and their carers. Nonpharmacol Ther Dementia. 2011a;2 (1):19-39.

14. Meiland FJM, Dröes RM, De Lange J, Vernooij-Dassen MJFJ, Van Tilburg W. Predictors of effective support in meeting centers for people with dementia and their carers. Nonpharmacol Ther Dementia. 2010;1(3):251-270.

15. Brooker D, Evans SC, Evans SB, et al. Evaluation of the implementation of the meeting centres support program in Italy, Poland, and the UK; exploration of the effects on people with dementia. Int J Geriatr Psychiatry. 2018;33(7):883-892. doi:10.1002/ gps.4865

16. Evans SB, Evans SC, Brooker D, et al. The impact of the implementation of the Dutch meeting centers support programme for family carers of people with dementia in Italy, Poland and UK: a cross country validation project. Aging Ment Health. 2018:1-11. doi:10.1080/13607863.2018.1544207.

17. Szcześniak D, Rymaszewska J, Saibene FL, et al. Meeting Centres Support Programme highly appreciated by people with dementia and caregivers: a European cross-country evaluation; (submitted).

18. CIZ Landelijke rapportages, CIZ Basisrapportage AWBZ [National reports, Basic report AWBZ]; 2013. Available from: https://www. werkhoezithet.nl/doc/kennisbank/basisrapportage_awbz.pdf. Accesssed 15 February, 2019.

19. Van Mierlo LD, Meiland FJM, Dröes RM. Dementelcoach: effect of telephone coaching on carers of community dwelling people with dementia. Int Psychogeriatr. 2012;24(2):212-222. doi:10.1017/ S1041610211001827

20. Dröes RM, Van der Roest HG, Hattink B, Meiland FJ Development and evaluation of the European STAR training portal: skilling and reskilling carers of people with dementia. Lecture at 23rd Alzheimer Europe conference: Living well in a dementia-friendly society; October 2013; St Julian's, Malta.

21. Hattink B, Meiland F, Van der Roest H, et al. Web-based STAR Elearning course increases empathy and understanding in dementia caregivers: results from a randomized controlled trial in the Netherlands and the United Kingdom. $J$ Med Internet Res. 2015;17 (10):e241. doi:10.2196/jmir.4025 
22. Van Rijn A, Meiland FJM, Dröes RM. Linking DemenTalent to Meeting Centers for people with dementia and their caregivers; a process analysis into facilitators and barriers in 12 Dutch Meeting centers. International Psychogeriatrics. submitted 2019. doi:10.3310/hta23120

23. Steffen AM, Gant JR. A telehealth behavioral coaching intervention for neurocognitive disorder family carers. Int $J$ Geriatr Psychiatry. 2016;31(2):195-203. doi:10.1002/gps.4312

24. Cohen J. Statistical Power Analysis for the Behavioral Sciences. Hillsdale NJ: L. Erlbaum Associates; 1988.

25. Reisberg B, Ferris S, De Leon MJ, Crook T. The global deterioration scale for assessment of primary degenerative Dementia. Am J Psychiatry. 1982;139:1136-1139. doi:10.1176/ajp.139.9.1136

26. Reynolds T, Thornicroft G, Abas M, et al. Camberwell assessment of need for the elderly (CANE). Development, validity and reliability. Br J Psychiatry. 2000;176:444-452.

27. Cuijpers P, Salden M. De 'plezierige activiteiten lijst' voor ouderen. [The pleasant activities list for elderly]. Tijdschr Gerontol Geriatr. 1996;27:54-61.

28. Rosenberg M. Society and the Adolescent Self-image. Princeton, NJ: Princeton University Press; 1965.

29. Cummings JL, Mega M, Gray K, Rosenberg-Thompson S, Carusi DA, Gornbein J. The Neuro-psychiatric Inventory: comprehensive assessment of psychopathology in dementia. Neurology. 1994;44:2308-2314. doi:10.1212/wnl.44.12.2308

30. Meiland FJM, Dröes RM. Experienced Autonomy-scale. Amsterdam: Department of Psychiatry, VU University medical Center; 2010.

31. Brod M, Stewart AL, Sands L, Walton P. Conceptualization and measurement of quality of life in dementia: the Dementia Quality of Life instrument (DQoL). Gerontologist. 1999;39:25-35. doi:10.1093/geront/39.1.25

32. Logsdon RG, Gibbons LE, McCurry SM, Teri L. Quality of life in Alzheimer's disease: patient and caregiver reports. $J$ Ment Health Aging. 1999;5:21-32.

33. Moniz-Cook E, Vernooij-Dassen M, Woods R, et al.; for the Interdem group. A European Consensus on outcome measures for psychosocial intervention research in dementia care. Aging Ment Health. 2008;12 (1):14-29. doi:10.1080/13607860801919850

34. Kat MG, Jonghe JFM, De, Aalten P, Kalisvaart CJ, Dröes RM, Verhey FRJ. Neuropsychiatrische symptomen bij dementie: psychometrische aspecten van de Nederlandse Neuropsychiatric Inventory (NPI). Tijdschr Gerontol Geriatr. 2002;33:150-155.

35. de Jonge JF, Kat MG, Kalisvaart CJ, Boelaarts L. Neuropsychiatric Inventory Questionnaire (NPI-Q); a validity study of the Dutch form. Tijdschr Gerontol Geriatr. 2003;34(2):74-77.

36. Vernooij-Dassen MJ, Felling AJ, Brummelkamp E, Dauzenberg MG, Van Den Bos GA, Grol R. Assessment of caregiver's competence in dealing with the burden of caregiving for a dementia patient: a Short Sense of Competence Questionnaire (SSCQ) suitable for clinical practice. J Am Geriatr Soc. 1999;47:256-257.

37. Dröes RM, Meiland FJM, Schmitz M, van Tilburg W. An evaluation of the Meeting Centres Support Programme among persons with dementia and their carers. Nonpharmacol Ther Dementia. 2011;2(1):19-39.

38. Testad I, Corbett A, Aarsland D, et al. The value of personalized psychosocial interventions to address behavioral and psychological symptoms in people with dementia living in care home settings: a systematic review. Int Psychogeriatr. 2014;26(7):1083-1098. doi:10.1017/S1041610214000131
39. Scales K, Zimmerman S, Miller S. Evidence-based nonpharmacological practices to address behaviural and psychological symptoms of Dementia. Gerontologist. 2018;58(S1):S88-S102. doi:10.1093/geront/gnx167

40. Gitlin LN, Hauck WW, Dennis MP, Winter L. Maintenance of effects of the home environmental skill-building program for family caregivers and individuals with Alzheimer's disease and related disorders. J Gerontol A Biol Sci Med Sci. 2005;60(3):368-374. doi:10.1093/ gerona/60.10.1246

41. Graff MJ, Vernooij-Dassen MJ, Thijssen M, Dekker J, Hoefnagels WH, Olderikkert MG. Effects of community occupational therapy on quality of life, mood, and health status in dementia patients and their caregivers: a randomized controlled trial. J Gerontol A Biol Sci Med Sci. 2007;62(9):1002-1009. doi:10.1093/gerona/62.9.1002

42. Clare L, Linden DE, Woods RT, et al. Goal-oriented cognitive rehabilitation for people with early-stage Alzheimer disease: A single-blind randomized controlled trial of clinical efficacy. Am J Geriatr Psychiatry. 2010;18(10):928-939. doi:10.1097/JGP.0b013e3181d5792a

43. Van Haeften-van Dijk AM, Meiland FJM, Hattink BJJ, Bakker TJEM, Dröes RM. Community day care with carer support versus usual nursing home-based day care: effects on needs, behavior, mood, and quality of life of people with dementia. Int Psychogeriatr. 2016;28(4):631-645. doi:10.1017/S1041610215001921

44. Dröes RM, Van Mierlo LD, Meiland FJM, Van der Roest H. Memory problems in dementia: adaptation and coping strategies, and psychosocial treatments. Expert Rev Neurother. 2011b;11(12):1769-1782. doi:10.1586/ern.11.167

45. Park A-L. Is there anything special about intergenerational approaches to older people with Dementia? A review. J Alzheimers Dis Parkinsonism. 2014;4:172. doi:10.4172/2161-0460.1000172

46. De Bruin SR, Oosting SJ, Kuin Y, et al. Green care farms promote activity among elderly people with dementia. $J$ Hous Elderly. 2009;23(4):368-389. doi:10.1080/02763890903327275

47. Hendriks I, Meiland FJM, Gerritsen DL, Dröes RM. Implementation and impact of unforgettable: an interactive art program for people with dementia and their caregivers. Int Psychogeriatr. 2019;31 (3):351-362. doi:10.1017/S1041610218000959

48. Dröes RM, Chattat R, Diaz A, et al. Social health and Dementia. A European consensus on the operationalisation of the concept and directions for research and practice. Aging Ment Health. 2017;21 (1):4-17. doi:10.1080/13607863.2016.1254596

49. Fazio S, Pace D, Flinner J, Kallmyer B. The fundamentals of personcentered care for individuals with dementia. Gerontologist. 2018;58 (suppl_1):S10-S19. doi:10.1093/geront/gnx122

50. Gilhooly KJ, Gilhooly MLM, Sullivan MP, et al. A meta-review of stress, coping and interventions in dementia and dementia caregiving. BMC Geriatr. 2016;16:106. doi:10.1186/s12877-016$0280-8$

51. Jennings LA, Ramirez KD, Hays RD, Wenger NS, Reuben DB. Personalized goal attainment in Dementia care: measuring what persons with Dementia and their Caregivers Want. $J$ Am Geriatr Soc. 2018;66:2120-2127. doi:10.1111/jgs.15541

52. Van Rijn A, Meiland FJM, Dröes RM. Linking two new e-health caregiver interventions in to meeting centres for people with dementia and their carers; a process evaluation. Aging \& Mental Health. EPub 23 May 2019. doi:10.1080/13607863.2019.1617243. 


\section{Publish your work in this journal}

Clinical Interventions in Aging is an international, peer-reviewed journal focusing on evidence-based reports on the value or lack thereof of treatments intended to prevent or delay the onset of maladaptive correlates of aging in human beings. This journal is indexed on PubMed Central, MedLine, CAS, Scopus and the Elsevier
Bibliographic databases. The manuscript management system is completely online and includes a very quick and fair peer-review system, which is all easy to use. Visit http://www.dovepress.com/ testimonials.php to read real quotes from published authors.

Submit your manuscript here: https://www.dovepress.com/clinical-interventions-in-aging-journal 